\title{
CHEMICAL CARTOGRAPHY WITH APOGEE: LARGE-SCALE MEAN METALLICITY MAPS OF THE MILKY WAY DISK
}

\author{
Michael R. Hayden ${ }^{1}$, Jon A. Holtzman ${ }^{1}$, Jo Bovy ${ }^{2,24}$, Steven R. Majewski $^{3}$, Jennifer A. Johnson ${ }^{4}$, \\ Carlos Allende Prieto ${ }^{5,6}$, Timothy C. Beers ${ }^{7}$, Katia Cunha ${ }^{8,9}$, Peter M. Frinchaboy ${ }^{10}$, Ana E. García Pérez ${ }^{3}$, \\ Léo Girardi $^{11,12}$, Fred R. Hearty ${ }^{13}$, Young Sun Lee ${ }^{1}$, David Nidever ${ }^{14}$, Ricardo P. Schiavon ${ }^{15}$, \\ Katharine J. Schlesinger ${ }^{16}$, Donald P. Schneider ${ }^{12,17}$, Mathias Schultheis ${ }^{18}$, Matthew Shetrone ${ }^{19}$, \\ Verne V. Smith ${ }^{9,20}$, Gail Zasowski ${ }^{21}$, Dmitry Bizyaev ${ }^{22}$, Diane Feuillet ${ }^{1}$, Sten Hasselquist $^{1}$, Karen Kinemuchi ${ }^{22}$, \\ Elena Malanushenko ${ }^{22}$, Viktor Malanushenko ${ }^{22}$, Robert O' Connell $^{3}$, Kaike Pan ${ }^{22}$, and Keivan Stassun ${ }^{23}$ \\ ${ }^{1}$ New Mexico State University, Las Cruces, NM 88003, USA; mrhayden@nmsu.edu, holtz@nmsu.edu, yslee@nmsu.edu, feuilldk@nmsu.edu, sten@nmsu.edu \\ ${ }^{2}$ Institute for Advanced Study, Einstein Drive, Princeton, NJ 08540, USA; bovy@ias.edu \\ ${ }^{3}$ Department of Astronomy, University of Virginia, Charlottesville, VA 22904-4325, USA; srm4n@ virginia.edu, aeg4x@ virginia.edu, rwo@virginia.edu \\ ${ }^{4}$ Department of Astronomy, The Ohio State University, Columbus, OH 43210, USA; jaj@ astronomy.ohio-state.edu \\ ${ }^{5}$ Instituto de Astrofísica de Canarias, E-38205 La Laguna, Tenerife, Spain; callende@iac.es \\ ${ }^{6}$ Departamento de Astrofísica, Universidad de La Laguna, E-38206 La Laguna, Tenerife, Spain \\ ${ }^{7}$ Department of Physics and Astronomy and JINA, Joint Institute for Nuclear Astrophysics, \\ Michigan State University, E. Lansing, MI 48824, USA; beers@pa.msu.edu \\ ${ }^{8}$ Observatório Nacional, São Cristóvão, Rio de Janeiro, Brazil; cunha@email.noao.edu \\ ${ }^{9}$ Steward Observatory, University of Arizona, Tucson, AZ 85721, USA \\ ${ }^{10}$ Texas Christian University, Fort Worth, TX 76129, USA; p.frinchaboy@ tcu.edu \\ ${ }_{11}$ Osservatorio Astronomico di Padova-INAF, Vicolo dell’Osservatorio 5, I-35122 Padova, Italy; leo.girardi@oapd.inaf.it \\ ${ }^{12}$ Laboratório Interinstitucional de e-Astronomia-LIneA, Rua Gal. José Cristino 77, Rio de Janeiro, RJ - 20921-400, Brazil \\ ${ }^{13}$ Department of Astronomy and Astrophysics, The Pennsylvania State University, University Park, PA 16802, USA; frh10@psu.edu, dps7@psu.edu \\ ${ }^{14}$ Department of Astronomy, University of Michigan, Ann Arbor, MI 48109, USA; dnidever@umich.edu \\ ${ }^{15}$ Astrophysics Research Institute, Liverpool John Moores University, Twelve Quays House, Egerton Wharf, Birkenhead CH41 ILD, UK; rpschiavon@ @mail.com
${ }_{16}$ Research School of Astronomy and Astrophysics, Australian National University, Mt. Stromlo Observatory, \\ Cotter Road, Weston, ACT 2611, Australia; katharine.schlesinger@anu.edu.au
${ }^{17}$ Institute for Gravitation and the Cosmos, The Pennsylvania State University, University Park, PA 16802, USA \\ ${ }^{18}$ Observatoire de la Cote d'Azur, Boulevard de l’Observatoire, B.P. 4229, F-06304 NICE Cedex 4, France; mathias.schultheis@oca.eu \\ ${ }^{19}$ University of Texas at Austin, McDonald Observatory, Fort Davis, TX 79734, USA; shetrone@ astro.as.utexas.edu \\ ${ }^{20}$ National Optical Astronomy Observatories, Tucson, AZ 85719, USA; vsmith@email.noao.edu \\ ${ }^{21}$ Department of Physics and Astronomy, Johns Hopkins University, Baltimore, MD 21218, USA; gail.zasowski@gmail.com \\ ${ }^{22}$ Apache Point Observatory, P.O. Box 59, Sunspot, NM 88349-0059, USA; dmbiz@apo.nmsu.edu, \\ kinemuchi@apo.nmsu.edu, elenam@apo.nmsu.edu, viktorm@apo.nmsu.edu, kpan@apo.nmsu.edu \\ ${ }^{23}$ Department of Physics and Astronomy, Vanderbilt Universiy, 6301 Stevenson Center, \\ VU Station B \#351807, Nashville, TN 37235, USA; keivan.stassun@ vanderbilt.edu \\ Received 2013 November 6; accepted 2014 January 23; published 2014 April 11
}

\begin{abstract}
We present Galactic mean metallicity maps derived from the first year of the SDSS-III APOGEE experiment. Mean abundances in different zones of projected Galactocentric radius $(0<R<15 \mathrm{kpc})$ at a range of heights above the plane $(0<|z|<3 \mathrm{kpc})$, are derived from a sample of nearly 20,000 giant stars with unprecedented coverage, including stars in the Galactic mid-plane at large distances. We also split the sample into subsamples of stars with low- and high- $[\alpha / \mathrm{M}]$ abundance ratios. We assess possible biases in deriving the mean abundances, and find that they are likely to be small except in the inner regions of the Galaxy. A negative radial metallicity gradient exists over much of the Galaxy; however, the gradient appears to flatten for $R<6 \mathrm{kpc}$, in particular near the Galactic mid-plane and for low- $[\alpha / \mathrm{M}]$ stars. At $R>6 \mathrm{kpc}$, the gradient flattens as one moves off the plane, and is flatter at all heights for high- $[\alpha / \mathrm{M}]$ stars than for low- $[\alpha / \mathrm{M}]$ stars. Alternatively, these gradients can be described as vertical gradients that flatten at larger Galactocentric radius; these vertical gradients are similar for both low- and high- $[\alpha / \mathrm{M}]$ populations. Stars with higher $[\alpha / \mathrm{M}]$ appear to have a flatter radial gradient than stars with lower $[\alpha / \mathrm{M}]$. This could suggest that the metallicity gradient has grown steeper with time or, alternatively, that gradients are washed out over time by migration of stars.
\end{abstract}

Key words: Galaxy: abundances - Galaxy: disk - Galaxy: stellar content - Galaxy: structure

Online-only material: color figures

\section{INTRODUCTION}

The variation of stellar chemical abundances within galaxies contains information about how galaxies are assembled, and tracks processes such as gas accretion, star formation timescales, and stellar migration. Within galaxy disks, measurements of

\footnotetext{
${ }^{24}$ Hubble Fellow.
}

abundance gradients have the potential to distinguish between different models of disk formation. Many models predict that galaxies form inside-out, with star formation proceeding more rapidly, and perhaps starting earlier, in the inner regions of disks (e.g., Larson 1976; Kobayashi \& Nakasato 2011; Bird et al. 2013). Models of chemical and dynamical evolution are becoming increasingly sophisticated, and it is now recognized that the structure of disks likely evolves with time. Chemical 
evolution models from Hou et al. (2000) predict that radial metallicity gradients arise naturally in the disk and flatten with time, while models from Chiappini et al. (2001) predict that metallicity gradients present in the disk will steepen with time. Recent models and simulations postulate that migration of stars within disks may be important to understanding the observed chemical structure of the disk (e.g., Sellwood \& Binney 2002; Schönrich \& Binney 2009; Loebman et al. 2011; Bird et al. 2013; Kubryk et al. 2013). Observations of chemical abundance gradients for a range of populations of stars are critical to our understanding of the evolution of the Milky Way.

The Milky Way provides the opportunity to measure large numbers of stellar abundances on a star-by-star basis, and chemical abundance gradients in different elements have been observed in the Milky Way disk in both the radial and vertical directions. Various tracers have been used including Cepheid variables (e.g., Luck et al. 2011; Lemasle et al. 2013), planetary nebulae (e.g., Henry et al. 2010; Stanghellini \& Haywood 2010), H II regions (e.g., Balser et al. 2011), open clusters (e.g., Carrera \& Pancino 2011; Frinchaboy et al. 2013), B stars (e.g., Daflon \& Cunha 2004; Daflon et al. 2009), and surveys of main sequence stars (e.g., Cheng et al. 2012b for the Sloan Extension for Galactic Understanding and Exploration (SEGUE; Yanny et al. 2009) of the Sloan Digital Sky Survey (SDSS; York 2000); Nordström et al. 2004 for the GenevaCopenhagen Survey (GCS; Boeche et al. 2013) for the Radial Velocity Experiment (Steinmetz et al. 2006)). Near the solar circle, the measured amplitude of the Milky Way radial gradient ranges from $-0.04 \mathrm{dex} \mathrm{kpc}^{-1}$ in $[\mathrm{S} / \mathrm{H}]$ in OB stars (Daflon et al. 2009) to $-0.099 \mathrm{dex} \mathrm{kpc}^{-1}$ in $[\mathrm{Fe} / \mathrm{H}]$ in main sequence stars between $4<$ Gyr $<6$ from the GCS (Nordström et al. 2004). Frinchaboy et al. (2013) measure a slope of -0.09 dex $\mathrm{kpc}^{-1}$ in $[\mathrm{M} / \mathrm{H}]$, and find a roughly flat gradient in $[\alpha / \mathrm{M}]$ for their sample of open clusters.

The behavior of the radial gradient with location has also been found to vary with different populations. Open cluster measurements (Yong et al. 2012; Frinchaboy et al. 2013) have found a flattening of the radial gradient at large Galactocentric radii, while observations with Cepheid variables (Luck \& Lambert 2011; Lemasle et al. 2013) find that the slope does not change in the outer disk. In the inner Galaxy, Henry et al. (2010) find that the slope of the radial gradient is shallower than at the solar circle using planetary nebula, but Cepheid observations (Luck \& Lambert 2011; Genovali et al. 2013) find that the gradient is steepest in the inner Galaxy. These previous studies have generally been limited in sample size to less than a few hundred objects, and generally target objects close to the plane of the Galaxy.

To the extent to which different tracers probe populations of different ages, comparisons of measured metallicity gradients have the potential to provide information on the evolution of chemical gradients with time. However, there is still disagreement on whether the observed gradients steepen or flatten with time, with some studies finding the radial gradient steepens with time (Stanghellini \& Haywood 2010), and others reporting that the gradient has flattened with time (Maciel \& Costa 2009). Recent models and simulations that include radial migration predict that observed gradients will be flatter for older populations, as migration washes out the gradients of older populations (e.g., Roškar et al. 2008; Loebman et al. 2011; Kubryk et al. 2013). Direct comparison between different samples still remains problematic; a uniform sample spanning a range of ages is needed to help address these ongoing questions. There may also be differences in gradients as measured by different elements, further complicating interpretation if different elements are measured in different tracer populations.

Vertical metallicity gradients have also been characterized near the solar circle (e.g., Hartkopf \& Yoss 1982; Chen et al. 2003, 2011; Marsakov \& Borkova 2006) for both thin- and thick-disk populations, although there is a range in reported slopes. The slope of the vertical gradient has been measured to be $-0.22 \mathrm{dex} \mathrm{kpc}^{-1}$ (Ak et al. 2007) for $|z|<3 \mathrm{kpc}$ in a sample of high-latitude $\mathrm{G}$ dwarfs from DR5 (Adelman-McCarthy et al. 2007) of the SDSS, $-0.22 \mathrm{dex} \mathrm{kpc}^{-1}$ in a sample of horizontalbranch stars (Chen et al. 2011) from DR8 (Aihara et al. 2011), and $-0.14 \mathrm{dex} \mathrm{kpc}^{-1}$ from a sample of kinematically selected thick-disk F/G/K dwarfs (Kordopatis et al. 2011). For samples targeting stars closer to the disk, Chen et al. (2003) measure a slope of $-0.295 \mathrm{dex} \mathrm{kpc}^{-1}$ using open clusters, Bartašiūtè et al. (2003) obtain $-0.23 \mathrm{dex} \mathrm{kpc}^{-1}$ for a sample of thin-disk stars identified by kinematics and asymmetric drift, Marsakov $\&$ Borkova (2006) find a slope of $-0.29 \mathrm{dex} \mathrm{kpc}^{-1}$ for thin-disk stars selected based on chemistry and orbital parameters, and Soubiran et al. (2008) measure a slope of $-0.31 \mathrm{dex} \mathrm{kpc}^{-1}$ for $|z|<1 \mathrm{kpc}$ from a sample of red clump giants.

The structure of the disk is also reflected in detailed abundances, with stars at greater distances from the plane typically having higher $[\alpha / \mathrm{Fe}]$ ratios than stars closer to the plane (e.g., Lee et al. 2011; Bovy et al. 2012c; Schlesinger et al. 2012). The nature of the vertical structure continues to be debated, with some studies suggesting that the thin and thick disks are distinct populations, while others report that there is just a single component with a continuous distribution of properties (e.g., Ivezic et al. 2008; Bovy et al. 2012a, 2012b, 2012c). Stars at larger distances from the plane have been observed to have flat or slightly positive radial metallicity gradients (Allende Prieto et al. 2006; Jurić et al. 2008; Carrell et al. 2012; Cheng et al. 2012b), as have older stars closer to the plane (Nordström et al. 2004). The flattening of the radial gradient with height might be explained by a transition between the thin- and thick-disk populations; Jurić et al. (2008) estimate that the thick disk becomes the dominant population at about $1 \mathrm{kpc}$ above the Galactic plane, which is where the radial metallicity gradients have been observed to be flat.

Models that attempt to explain the properties of the disk can be better constrained with metallicity information covering a wide range of the Galactic disk. In particular, the ability of radial migration models to explain the scatter in the local population depends on the metallicity distribution function (MDF) in the inner Galaxy. Whether radial mixing can heat stars enough to result in a thick-disk population with the vertical scale height of the Milky Way's thick disk is the subject of much theoretical dispute (e.g., Minchev et al. 2012). Observations that provide information on the distribution of metallicities through the entire disk can provide strong observational constraints, and observations of detailed abundances might make it possible to identify the birthplace of different populations of stars.

Measuring chemical abundances in the plane of the disk for a sample covering a large range of Galactocentric radii is challenging because of the significant extinction within the disk. The Apache Point Observatory Galactic Evolution Experiment (APOGEE; Allende Prieto et al. 2008) of the Sloan Digital Sky Survey-III (SDSS-III; Eisenstein et al. 2011) provides a unique opportunity to map the chemistry of stars in all Galactic zones, because it obtains spectra in the near-IR, where the effects of extinction are reduced. APOGEE is a high-resolution 


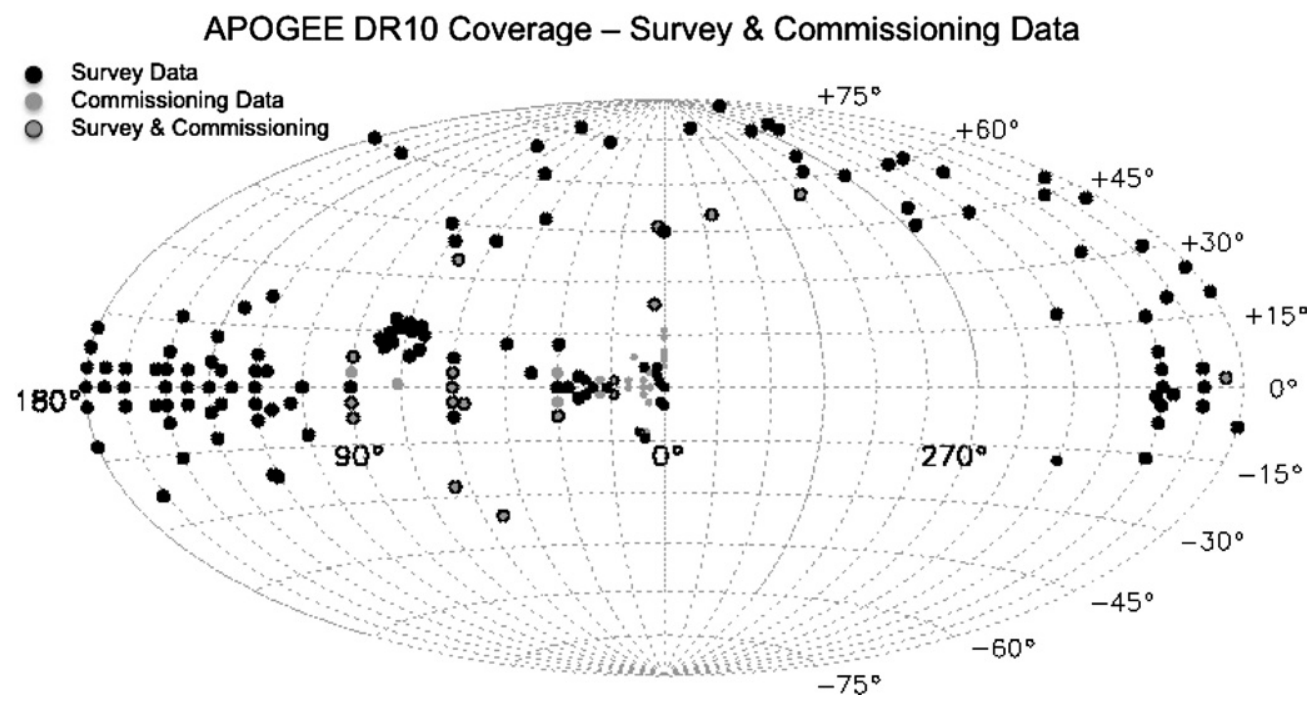

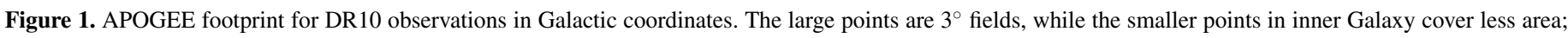

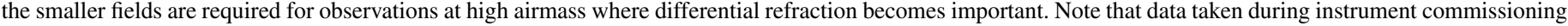
are of slightly degraded quality and are not used in this paper.

( $R \sim 22,500)$ spectrograph that records stellar spectra in the $H$ band between 1.51 and $1.70 \mu \mathrm{m}$ using the SDSS $2.5 \mathrm{~m}$ telescope (Gunn et al. 2006). The APOGEE survey aims to obtain high signal-to-noise ratio $(\mathrm{S} / \mathrm{N} \sim 100)$ spectra of $10^{5}$ stars over three years of operation, with the majority of these targets being red giants. Because of the intrinsic luminosity of giants and the significant reduction in extinction in the $H$ band relative to the optical, APOGEE is capable of observing stars directly in the plane of the Galaxy to large distances. As a result, the survey provides coverage over a large range of Galactocentric radii and enables the characterization of stellar abundances across the Galaxy. The eventual goal is to determine individual elemental abundances for $\sim 15$ different elements for the bulk of the survey stars.

Having a sample of tens of thousands of objects covering the Galaxy from the bulge to the edge of the disk, at a large range of heights about the plane, combined with accurate chemical abundances for up to 15 elements, makes APOGEE unique in its ability to study the Milky Way. Previous studies of the radial gradient used bright tracers that lie in the plane of the Galaxy (e.g., Cepheids (Luck et al. 2011), H II regions (Balser et al. 2011)), which have good radial coverage from the bulge to the outer disk, but lack vertical coverage and have small sample sizes. Studies with larger sample sizes, such as largescale surveys like SEGUE (Cheng et al. 2012b) or the GCS (Nordström et al. 2004), were done with observations taken in the optical regime and have limited radial coverage in the plane of the Galaxy. The vertical gradient has only been characterized near the solar circle (e.g., Ak et al. 2007; Chen et al. 2011; Kordopatis et al. 2011), and has not been measured at other locations in the disk. APOGEE is the first survey that provides a large sample with excellent spatial coverage of the Galaxy, allowing the simultaneous determination of radial and vertical gradients across the disk.

In this paper, we present results on mean abundances in the disk using data from the first year of the APOGEE survey. Our results focus on giant stars with projected Galactocentric radii $(R)$ between $0<R<15 \mathrm{kpc}$ and with $0<|z|<2 \mathrm{kpc}$, and we restrict our discussion primarily to the overall metal abundance, although we use the derived $[\alpha / \mathrm{M}]$ ratios to define low- $[\alpha / \mathrm{M}]$ and high- $[\alpha / \mathrm{M}]$ subsamples. A complementary paper (Anders et al. 2013) presents results from a subsample of the first year data for which three-dimensional kinematics can be derived. Discussion of other elemental abundances is deferred to future work, as the ability to derive these abundances is still being developed.

\section{DATA AND SAMPLE SELECTION}

Data are taken from the Tenth Data Release of the Sloan Digital Sky Survey (DR10; Ahn et al. 2013), which contains stellar spectra and derived stellar parameters for stars observed during the first year of SDSS-III/APOGEE. These stars cover a wide range of the Galaxy (Figure 1), and span a range of magnitudes between $8<H<13.8$ for primary science targets. Target selection is described in detail in Zasowski et al. (2013). To summarize briefly, the sample is selected from the Two Micron All Sky Survey (2MASS) catalog using a dereddened color cut of $(J-K)_{0}>0.5$ to remove hotter main sequence stars and to ensure that only stars with $T_{\text {eff }} \lesssim 5500 \mathrm{~K}$ (for which stellar parameters and abundances can be accurately determined) are selected, but without biasing the sample against metal-poor stars. The dereddening is accomplished using the Rayleigh-Jeans Color Excess method (RJCE; Majewski et al. 2011), which uses 2MASS photometry in conjunction with nearIR photometry from the Spitzer/IRAC (Fazio \& Team 2004) GLIMPSE surveys (Benjamin et al. 2003; Churchwell et al. 2009) where available or from WISE (Wright et al. 2010).

APOGEE is a fiber-fed spectrograph that records 300 spectra simultaneously. Standard fields include 230 science targets, 35 hot stars to characterize telluric absorption, and 35 sky fibers. Exposures of $500 \mathrm{~s}$ are obtained in a series of 47 non-destructive readouts throughout the exposure and are taken in pairs, with a 0.5 pixel detector shift between the two exposures to provide well-sampled spectra across a spectral range that runs from 1.51 to $1.70 \mu \mathrm{m}$; a typical visit includes four pairs of exposures. Typically, each field is visited at least three times to identify radial velocity variables, except for selected bulge fields.

Data are processed through a standard pipeline that performs basic calibrations and collapses the data cubes to twodimensional images, extracts spectra, measures radial velocities, and combines spectra from different visits (D. Nidever et al. in preparation). Stellar parameters and abundances are derived 
by the APOGEE Stellar Parameters and Chemical Abundances Pipeline (ASPCAP; A. Garcia Perez \& C. Allende Prieto (in preparation)), which determines the best-matching parameters by searching within a multi-dimensional grid of synthetic spectra. For DR10, the dimensions that are used to characterize the spectra include effective temperature $\left(T_{\text {eff }}\right)$, surface gravity $(\log g)$, overall metal abundance $([\mathrm{M} / \mathrm{H}]), \alpha$ element abundances $([\alpha / \mathrm{M}])$, carbon abundance $([\mathrm{C} / \mathrm{M}])$, and nitrogen abundance $([\mathrm{N} / \mathrm{M}])$; the latter three abundances are included as fundamental stellar parameters because of the strong importance of molecular features from $\mathrm{CN}, \mathrm{CO}$, and $\mathrm{OH}$ in the region covered by the APOGEE spectrum. The current grid of synthetic spectra covers $T_{\text {eff }}>3500 \mathrm{~K}$ and $\log g>0$; stars with derived parameters that fall close to the grid edges are flagged as likely to be unreliable.

Additional details on sample selection and data processing are provided in the APOGEE targeting paper (Zasowski et al. 2013), the DR10 paper (Ahn et al. 2013), and on the DR10 Web site. $^{25}$

The quality of the derived parameters and abundances has been discussed in Mészáros et al. (2013), based primarily on APOGEE observations of stars in clusters with known abundances. This study found small systematic offsets in some of the ASPCAP-derived parameters; these corrections have been applied in the DR10 data release and are used here. Results for dwarfs $(\log g>3.8)$ were found to be less reliable, and insufficient calibrators are available for these objects, so we only include stars with $\log g<3.8$.

Mészáros et al. (2013) also characterize the uncertainties in derived parameters and abundances after calibration, and find that $[\mathrm{M} / \mathrm{H}]$ and $[\alpha / \mathrm{M}]$ typically have a precision of $0.1 \mathrm{dex}$, uncertainties in temperature are $\sim 100 \mathrm{~K}$, and uncertainties in $\log g$ are $\sim 0.2$ dex. However, there is some indication that the $[\alpha / \mathrm{M}]$ results are unreliable for cooler $\left(T_{\text {eff }}<4200 \mathrm{~K}\right)$ stars, as discussed further below. Results for carbon and nitrogen are considerably less certain and are still a topic of ongoing investigation.

\subsection{Distances}

Distances for each star are determined from the derived stellar parameters based on Bayesian statistics, following methods described by Burnett \& Binney (2010); see also Santiago et al. (2014). The probability of all possible distances is calculated for each star given the extinction-corrected magnitude (using RJCE, as referenced above) and the stellar parameters from ASPCAP, under the assumption that the parameters are related according to the theoretical isochrones of the Padova group (Bressan et al. 2012). The isochrones also account for the relative numbers of stars with different parameters as determined by the initial mass function and the timescales for different stages of stellar evolution. Density distributions of four Galactic components (thin disk, thick disk, triaxial bulge, and halo) are included as priors in computing the distance probability distribution function, as well as priors on the age distribution of each component. For each star, the mean distance, as determined from its distance probability distribution function, is adopted. Given distances, we calculate projected in-plane Galactocentric radius $(R)$ assuming that the Sun is located $8 \mathrm{kpc}$ from the Galactic center.

Distances were tested using a set of simulated observations from TRILEGAL (Girardi et al. 2005), observations of

\footnotetext{
25 http://www.sdss3.org/dr10
}

calibration clusters (Mészáros et al. 2013) of known distance, and observations of Kepler giants with asteroseismic parameters (M. Pinsonneault et al. in preparation) that allow independent distance estimates. Generally, we find distance errors of $\lesssim 20 \%$ for individual stars. The error in $R$ is a function of the error in the distance and the line-of-sight of the observation; errors in $R$ are generally smaller than the error in distance. Future additions to the priors, such as the incorporation of the APOGEE selection function and three-dimensional extinction maps (M. Schultheis et al. in preparation), are planned to help to improve the accuracy of distance estimates.

\subsection{Potential Metallicity Biases}

Before presenting mean metallicity results, we consider several potential sources of bias in our observed metallicities.

1. Bias from sample selection: color cut and magnitude limits.

2. ASPCAP spectral grid edges: spectral library has no results for $T_{\text {eff }}<3500 \mathrm{~K}$ and $\log g<0$.

3. Metallicity bias in derived distances.

\subsubsection{Biases from Sample and ASPCAP Selection}

We use a set of simulated observations from TRILEGAL that were run through our targeting selection scheme (color cut and magnitude distributions) and the DR10 selection of fields to determine the effect of target selection on the observed metallicities. These simulations include different MDFs for different Galactic components, but not any changes in the MDF of any individual component (i.e., no disk metallicity gradient). Nevertheless, these simulations are still useful for determining whether spatial variations might be introduced because of sampling effects that arise because the typical parameters of stars in different Galactic zones will be different (e.g., more distant stars will tend to be intrinsically more luminous). The simulations are for fields with $l<90^{\circ}$ (i.e., the inner Galaxy fields), where the effect of the ASPCAP grid edge will be the largest on the sample.

Results are shown in the top row of Figure 2 for several different Galactocentric radii, to illustrate sample selection effects only; there is little difference in the sampled MDFs relative to the underlying ones.

The second row shows the effect of limiting the TRILEGAL sample to $T_{\text {eff }}>3540 \mathrm{~K}$, to test the effects of the ASPCAP grid edge on the observed MDF. This constraint clearly leads to a bias against high-metallicity stars in more distant zones, as expected, because the giant branch is cooler at higher luminosity and metallicity. The extent to which such a bias exists in the real data depends on how many high-metallicity stars actually exist at these distances, but, since the effect is significant for $[\mathrm{M} / \mathrm{H}]>0.1$, this effect is likely to be an issue for the APOGEE survey. The overall number of stars that have ASPCAP parameters at the grid edges is small: only 300 stars out of our sample of 20,000. However, most of these objects are in the direction of the inner Galaxy. Because we cannot derive the stellar parameters for these stars, we are not able to determine their distance, but it is not impossible that most of them are located at small Galactocentric radii and could impact the observed mean metallicities in the inner Galaxy.

The bias against high-metallicity stars also appears in the simulations at large Galactocentric radii, but this is likely due to the fact that sampled simulations have only been made for $l<$ $90^{\circ}$, so all of the stars in the simulation at large Galactocentric radius are at very large distances from the Sun, and, as a result, 




Figure 2. Metallicity distribution function (MDF) for the entire input TRILEGAL simulation (red filled) vs. the MDF of various sample selections from the simulations (blue line), in five different radial bins from left to right. The top row (A) shows target selection only, the second row (B) shows additional limitation of $T_{\text {eff }}>3540 \mathrm{~K}$, the third row (C) shows restriction to $\log g>0$, the fourth row (D) shows $\log g>0.9$, and the fifth row (E) shows the combined effects of both the surface gravity and temperature restrictions. The fourth and fifth rows have the same MDF: the effect of the temperature grid edge on metallicity has been removed with the surface gravity cut.

(A color version of this figure is available in the online journal.)

are of very low surface gravity and temperature. In the actual APOGEE data, most of the stars at larger Galactocentric radii are in the direction of the anti-center, and thus at smaller distances from the Sun, so they are dominated by stars of higher surface gravity and temperature, for which the bias is less significant. Furthermore, there are very few stars near the grid edge in the outer regions.

The third row shows the effect of removing stars with $\log g<0.0625$, as also imposed by the limitations of the
ASPCAP grid. This effect is small, because there are few of these stars (less than $4 \%$ of the total simulated sample), and surface gravity is not as sensitive to metallicity as temperature.

The metallicity bias from the cool temperature limit can be alleviated by restricting the sample to stars of higher surface gravity, at the expense of preferentially removing more distant stars from the sample. This is demonstrated in the bottom panels of Figure 2, which show the MDFs for a restricted sample with $\log g>0.9$, both without and with a temperature cut 
of $T_{\text {eff }}>3540 \mathrm{~K}$. A small bias is present at larger distances, but it is significantly smaller than for the sample without a surface gravity cut, and mostly affects stars of higher metallicity $([\mathrm{M} / \mathrm{H}] \gtrsim 0.3)$. This bias is predominantly a concern for the inner Galaxy, where more metal-rich stars may exist in larger numbers. Existing measurements of bulge stars (e.g., Zoccali et al. 2008; Gonzalez et al. 2013; Ness et al. 2013) suggest that metal-rich stars are actually less prominent there than they are in the TRILEGAL simulations, so the bias in the actual data may be smaller than is present in our simulations. Future work (A. Garcia Perez et al. in preparation) will consider the MDF of the bulge as derived from APOGEE observations.

The simulations suggest that limiting the sample to $\log g>$ 0.9 avoids significant bias in the mean metallicities, although this does rest on the assumption that the MDF of the simulation is representative of the real Galaxy. In general, however, a viable test for biases can be made by measuring the extent to which the MDFs (or mean metallicities) change as the sample is restricted to stars of higher surface gravity.

\subsubsection{Biases from Distance Determination}

Our method of distance determination is not expected to have a strong sensitivity to metallicity, because the dominant parameter that constrains the distance is the observed surface gravity, and this depends only weakly on metallicity. We verified this expectation by recovering distances from a TRILEGAL simulation where errors in metallicity, surface gravity, and temperature were added according to our estimates of how accurately they are measured by APOGEE ( $100 \mathrm{~K}$ in $T_{\text {eff }}, 0.1$ dex in $[\mathrm{M} / \mathrm{H}]$, and $0.2 \mathrm{dex}$ in $\log g$ ). No significant trend in distance error is seen with the input error in metallicity. Instead, as expected, the distance errors are significantly correlated with the input error in surface gravity.

Another bias could arise from the use of expected density distributions for each Galactic component in our Galactic model priors. However, we did not include any assumption about the MDF of the different Galactic components in the priors that we used for the distance determination (i.e., the metallicity of a star did not preclude it from falling at any distance or belonging to a particular Galactic component). We have also verified that, qualitatively, the results are not significantly different when we remove the use of distance priors altogether for the bulk of the disk sample. An exception is for stars in the direction of the bulge, for which the density distribution of the prior drives the distances to larger values; while we present results of these stars in this paper, we restrict most of the discussion to stars with $R>3 \mathrm{kpc}$.

\section{RESULTS}

To determine mean metallicities, we use a sample that includes all stars from the APOGEE DR10 release that were targeted as part of the "main survey" (i.e., we did not include special targets; including stars in clusters, calibration stars, stars observed for ancillary programs, etc.), have $\log g>0$, have $\mathrm{S} / \mathrm{N}>80$ per pixel in the combined spectrum, and are not flagged as bad by the ASPCAP pipeline (which includes flagging stars near the grid edges as bad; see the DR10 Web documentation ${ }^{26}$ for more details). In addition, we consider only stars less than $3 \mathrm{kpc}$ from the Galactic mid-plane $(|z|)$. These selection criteria yield a sample of 19,662 giants.

\footnotetext{
26 http://www.sdss3.org/dr10/irspec/
}
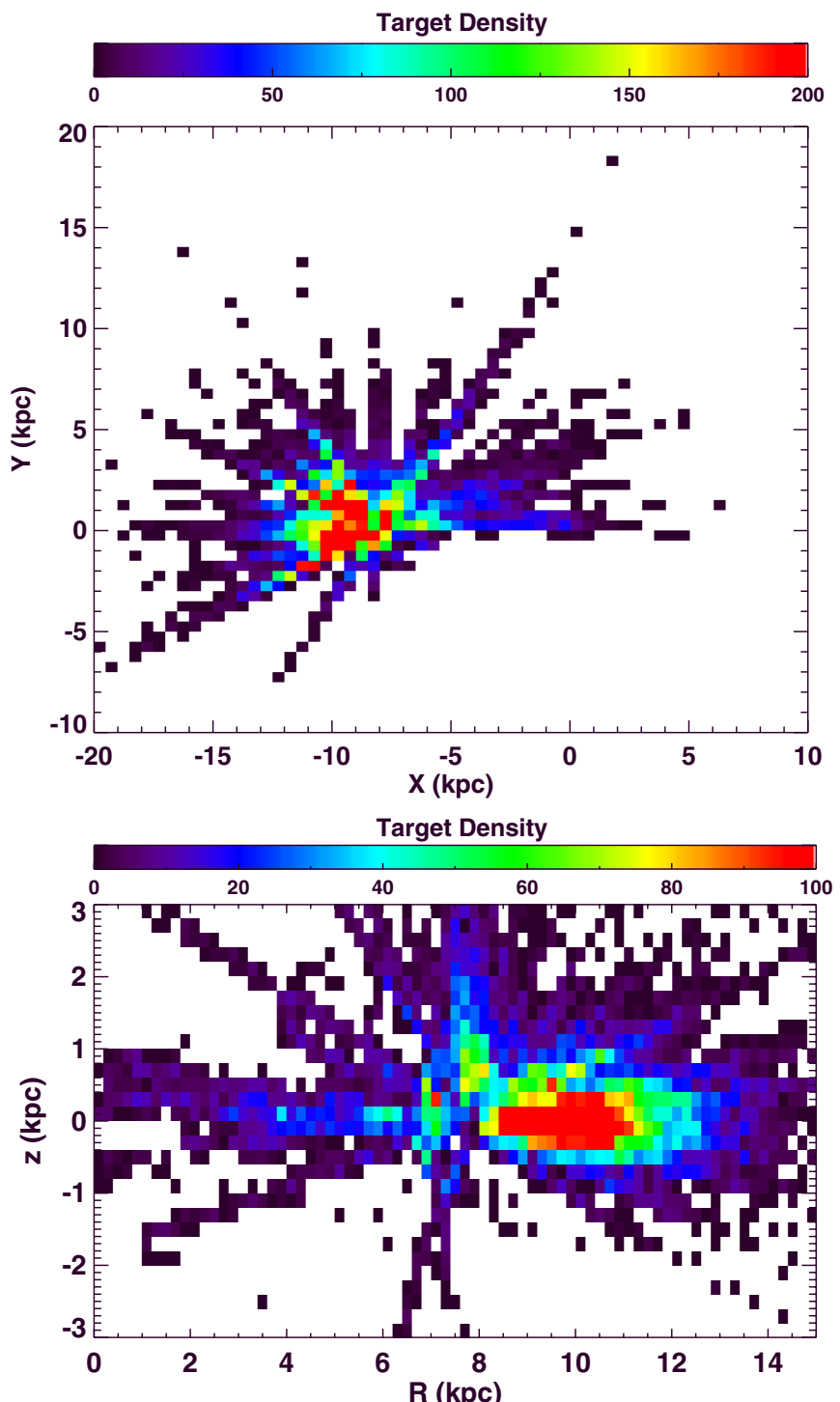

Figure 3. Top: a face-on view showing the stellar density in the APOGEE DR 10 sample for stars with $|z|<2 \mathrm{kpc}$ and $\log g>0.9$. The Galactic center is at $(0$, $0)$, while the Sun is located at $(-8,0 \mathrm{kpc})$. Bottom: the spatial density of targets in the $R-z$ plane. There are more targets above than below the plane, and more targets in the anti-center direction.

(A color version of this figure is available in the online journal.)

Figure 3 displays the observed number of stars in different Galactic zones for this sample. The top panel shows a face-on view of the Galactic disk, while the bottom panel shows an edge-on view in Galactocentric radius and distance from plane. The year 1 sample contains stars covering $0<R<15 \mathrm{kpc}$ and $-3<z<3 \mathrm{kpc}$ (mostly at $z>-1$ ) from the plane. There are significantly more stars in the anti-center direction because more fields were completed in that direction during the first year of observation.

To consider whether we are likely to have metallicity biases in the sample, the top panel of Figure 4 presents the difference between the mean metallicity of stars in the full sample to the mean metallicity of stars in a sample with $\log g>0.9$, and the bottom panel shows the ratio of a $\log g>0.9$ to $\log g>1.2$ sample, as a function of Galactocentric radius and distance from the plane. These results suggest that metallicity biases are significant only in the inner Galaxy, at $R<5 \mathrm{kpc}$ for the full sample, and that a sample with $\log g>0.9$ is not significantly 

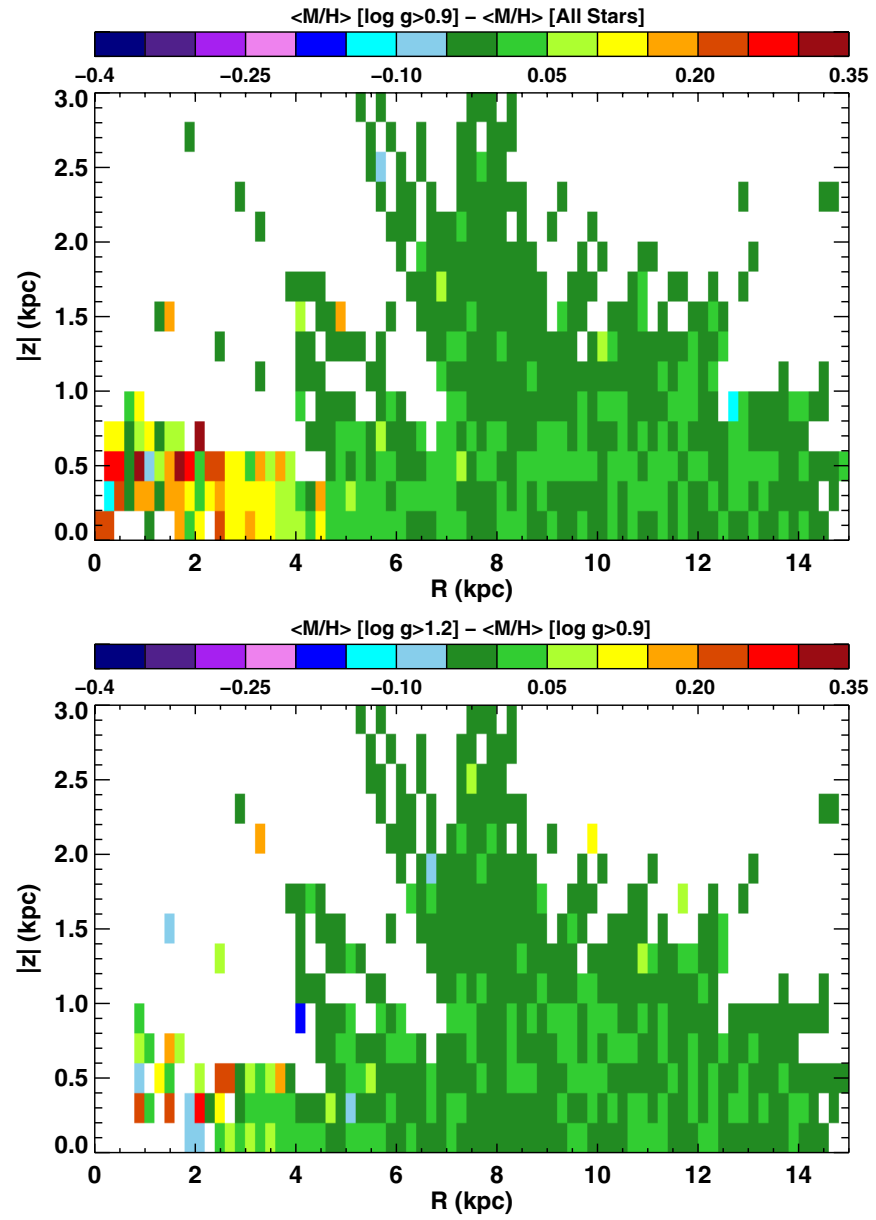

Figure 4. Top: difference in mean metallicity of the full sample compared to that of the $\log g>0.9$ sample. The difference in mean metallicity is large in the inner Galaxy $(R<4 \mathrm{kpc})$, which is where the bias from the ASPCAP grid edge impacts our results. Bottom: same as above, but between the $\log g>0.9$ and $\log g>1.2$ samples. The difference in metallicity between these samples is small, and generally less than $0.1 \mathrm{dex}$, implying that a more stringent surface gravity cut is not required to account for the metallicity bias introduced by the restriction to $T_{\text {eff }}>3540 \mathrm{~K}$.

affected by biases for $R \gtrsim 3 \mathrm{kpc}$. We adopt the $\log g>0.9$ cut for this paper, and future references to the data set include this cut unless otherwise noted.

While we include the data at $R<3 \mathrm{kpc}$ in our results, the main focus of this paper is on the disk at $R \gtrsim 3 \mathrm{kpc}$ because of the potential remaining biases in the inner regions, and also because our data contains relatively few stars in these regions. In addition, the inner regions may contain stars from structural components that might be distinct from the disk, i.e., the bulge and bar. We note that significant additional data were obtained for the inner Galaxy during APOGEE commissioning. These data are not included here because of our desire to work with a homogeneous sample; however, a future paper (A. Garcia Perez et al. in preparation) will discuss the bulge population, including results from commissioning data, in greater detail.

\subsection{Mean Metallicity Maps}

Our main result is shown in Figure 5. The top panel shows the mean metallicity of the $\log g>0.9$ sample in $0.2 \times 0.2 \mathrm{kpc}^{2}$ bins in Galactocentric radius and distance from the Galactic plane. In all zones, there is a substantial metallicity spread well

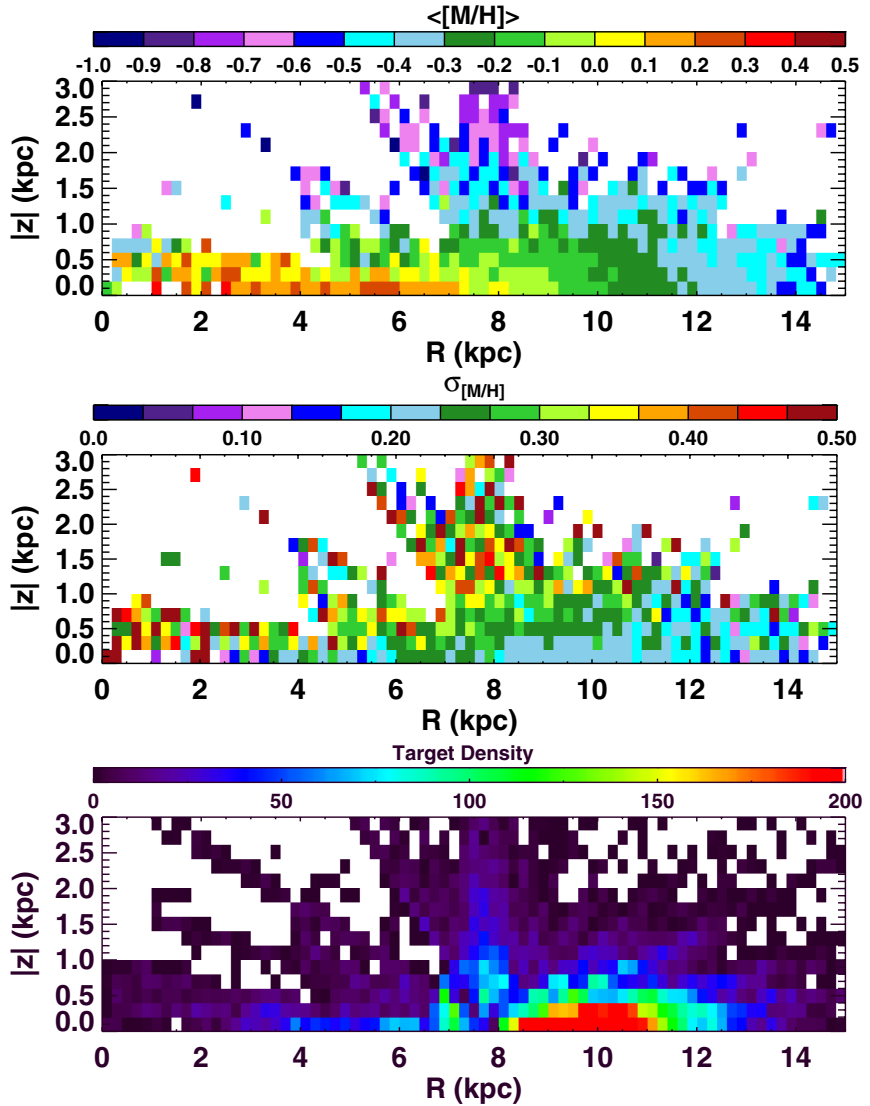

Figure 5. Top: the mean metallicity as a function of $R$ and $|z|$ for our sample of stars with $\log g>0.9$, in zones of $0.2 \times 0.2 \mathrm{kpc}^{2}$; a minimum of four stars is required to show a bin. The mean metallicity is roughly constant in the inner Galaxy close to the plane, and a negative gradient is clearly seen for populations with $R \gtrsim 6 \mathrm{kpc}$. The mean metallicity also decreases rapidly with height about the plane, and the radial gradients are less pronounced at larger heights about the plane. Middle: the standard deviation in metallicity at different locations in the Galaxy. The standard deviation at each location is much larger than the errors in metallicity for a single star. Bottom: the number of stars in each zone.

in excess of the expected abundance accuracy of 0.1 dex for an individual star; this is quantified in the central panel, which presents the standard deviation in each bin. The bottom panel gives the total number of stars in each bin.

Several features are immediately evident from Figure 5.

1. There are significant vertical metallicity gradients (see Section 3.3.1).

2. The vertical gradient is steeper in the inner regions of the Galaxy than in the outer regions (see Section 3.3.1).

3. The radial variation in mean metallicity decreases as one moves away from the plane (see Section 3.3.2).

4. In the plane, while the overall radial gradient is negative, it appears to flatten in the inner regions (see Section 3.3.2).

5. The spread in metallicity is everywhere larger than the uncertainty in the abundance determination; in the plane, the variation appears to be larger at smaller Galactocentric radius, and it also appears to increase as one moves above the plane.

APOGEE's red giant sample likely includes stars of a wide range of ages, so the observed metallicity map is almost certainly averaging over multiple populations of stars. In the case of a constant star formation rate where the distribution in stellar ages in the Galaxy is flat, isochrones suggest that the median age of giants is several Gyr, but depends on the luminosity of the giants: 
for red clump stars, the median age is $\sim 2$ Gyr (Girardi \& Salaris 2001), while for more luminous giants it is somewhat older. This implies that the mean metallicity is weighted toward larger ages for more distant zones-even if the star formation history was the same in different regions-and this could influence the interpretation of metallicity gradients from the entire sample. Variations in star formation history would further complicate the interpretation.

\section{2. $[\alpha / M]$ Subsamples}

As a first cut at separating the sample by age, we adopt $[\alpha / \mathrm{M}]$ as a potential age discriminator (Lee et al. 2011; Bensby et al. 2011; Cheng et al. 2012a; Bovy et al. 2012a, 2012b, 2012c), where stars with higher $[\alpha / \mathrm{M}]$ are more likely to be older stars. Unfortunately, the state of the ASPCAP pipeline used for the DR10 results has some potentially significant uncertainties with the determination of $[\alpha / \mathrm{M}]$. This is demonstrated in the top panel of Figure 6 , which shows $[\alpha / \mathrm{M}]$ as a function of $[\mathrm{M} / \mathrm{H}]$ for the APOGEE sample. Stars with an ASPCAP warning on $[\alpha / \mathrm{M}]$ are shown in red, and include all stars with $T_{\text {eff }}<4200 \mathrm{~K}$. The reason for the warning flag is apparent from the Figure: the cooler stars show a strong, narrow bimodal distribution in $[\alpha / \mathrm{M}]$ that is not seen at higher temperatures and is not seen by other more local studies. While one might imagine that this could potentially be an astrophysical effect-e.g., because the cooler stars are more luminous and thus more likely to be located at greater distances - we find that the bimodality persists even within stars in the same Galactic zone (middle panel of Figure 6). When the stars with $T_{\text {eff }}<4200 \mathrm{~K}$ are removed (bottom panel of Figure 6$)$, the observed $[\alpha / \mathrm{M}]$ distribution remains bimodal but with significant scatter, and resembles results obtained with smaller samples from the solar neighborhood (e.g., Adibekyan et al. 2012). We defer interpretation of the bimodal $[\alpha / \mathrm{M}]$ distribution (e.g., whether it is likely to be a selection effect or intrinsic to the Galaxy) to a later paper.

For the present purposes, we split the sample into low- and high- $[\alpha / \mathrm{M}]$ samples, along the line shown in Figure 6 . This split is similar to that used by Lee et al. (2011) for their SEGUE sample. We work with two high- $[\alpha / \mathrm{M}]$ samples, one including the stars with $T_{\text {eff }}<4200 \mathrm{~K}$ and one excluding them; while the $[\alpha / \mathrm{M}]$ results for the cooler stars are certainly suspect, it is not impossible that they can still distinguish between lower- and higher- $[\alpha / \mathrm{M}]$ stars. These cooler giants are critical for probing to larger distances, because they are intrinsically more luminous; certainly, however, results including these stars must be treated cautiously.

The subsamples contain 15,164 giants with $[\alpha / \mathrm{M}]$ abundances near solar, and 4,498 $[\alpha / \mathrm{M}]$-enhanced giants, if we include stars of all temperatures. If we remove stars with $T_{\text {eff }}<$ 4200 , we are left with sample of 14,150 low- $[\alpha / \mathrm{M}]$ and 3374 high- $[\alpha / \mathrm{M}]$ stars; the temperature cut affects the high- $[\alpha / \mathrm{M}]$ sample significantly more than the low one. In subsequent plots, we track three different $[\alpha / \mathrm{M}]$ subsamples: low- $[\alpha / \mathrm{M}]$, all high$[\alpha / \mathrm{M}]$, and high- $[\alpha / \mathrm{M}]$ with $T_{\text {eff }}>4200$.

Figure 7 presents the mean metallicity maps for the different $[\alpha / \mathrm{M}]$ subsamples: the upper panel contains the low- $[\alpha / \mathrm{M}]$ stars, while the middle and bottom panels show the high- $[\alpha / \mathrm{M}]$ stars without and with the low temperature limit, respectively. The high- $[\alpha / \mathrm{M}]$ stars are clearly more metal poor, and extend significantly higher above the mid-plane of the Galaxy; there are very few low- $[\alpha / \mathrm{M}]$ stars at $|z|>2 \mathrm{kpc}$. Lower- $[\alpha / \mathrm{M}]$ stars extend farther out in Galactocentric radius; there are very few high- $[\alpha / \mathrm{M}]$ stars beyond $R \gtrsim 12 \mathrm{kpc}$. There is a suggestion
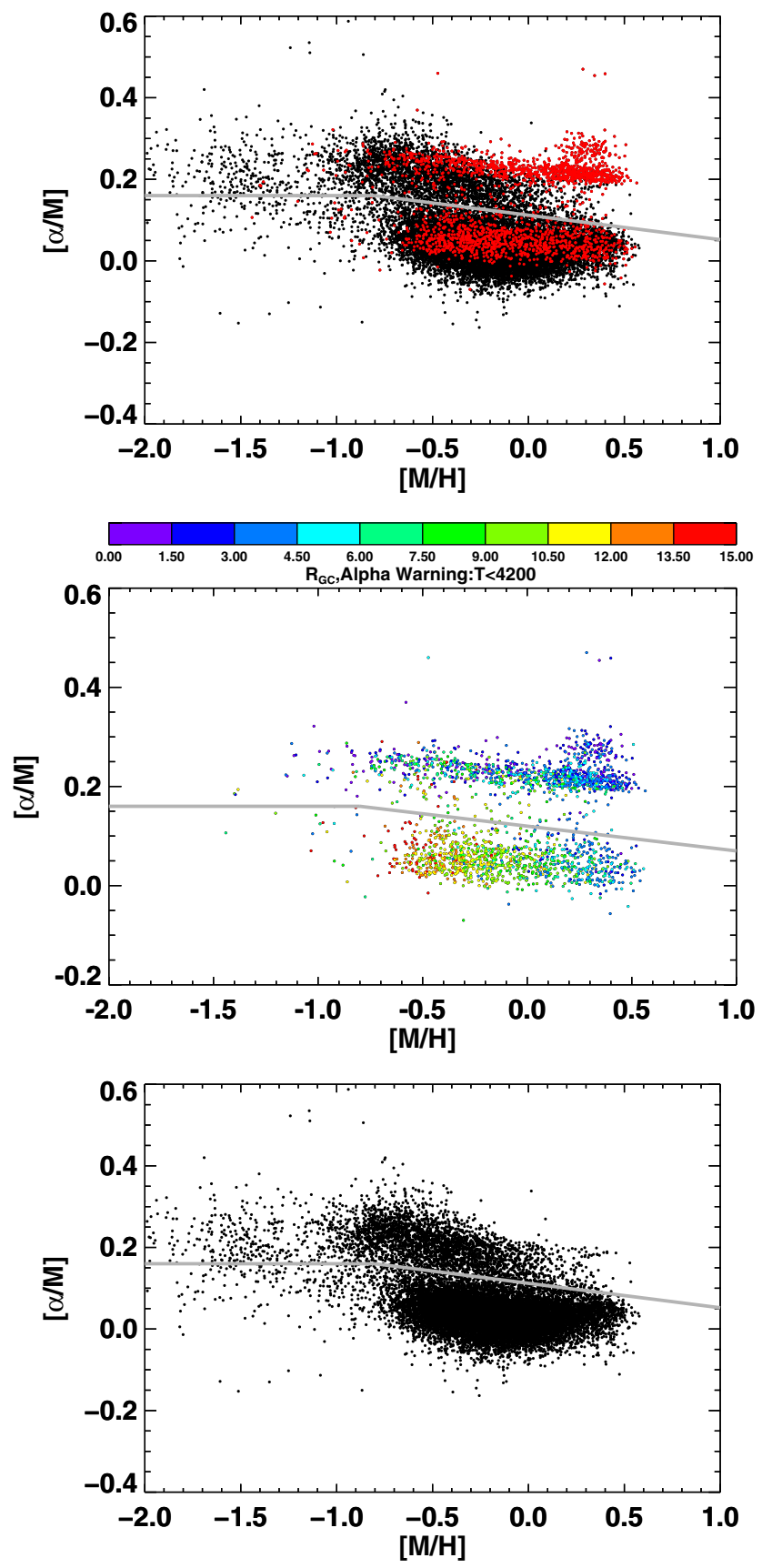

Figure 6. Top: the APOGEE DR 10 sample in $[\alpha / \mathrm{M}]$ vs. $[\mathrm{M} / \mathrm{H}]$ space. Stars with potentially inaccurate $[\alpha / \mathrm{M}]$ abundance determinations $\left(T_{\text {eff }}<4200 \mathrm{~K}\right)$ are shown in red. Middle: the $[\mathrm{M} / \mathrm{H}]$ vs. $[\alpha / \mathrm{M}]$ plot for the stars with $T_{\mathrm{eff}}<4200 \mathrm{~K}$, color coded by their derived Galactocentric radius. The bimodality is still present even for stars in the same areas of the Galaxy, suggesting that it is likely spurious. Bottom: the $[\mathrm{M} / \mathrm{H}]$ vs. $[\alpha / \mathrm{M}]$ relation for stars with $T_{\text {eff }}>4200 \mathrm{~K}$. In all panels, the gray line shows our separation of low- and high- $[\alpha / \mathrm{M}]$ abundance populations.

(A color version of this figure is available in the online journal.)

that the metallicity gradient for the low- $[\alpha / \mathrm{M}]$ stars flattens in the inner regions of the disk. In the outer Galaxy, the gradient in high- $[\alpha / \mathrm{M}]$ stars is significantly flatter than that in low- $[\alpha / \mathrm{M}]$ stars.

\subsection{Quantitative Gradients}

To quantify the observed gradients we measure the vertical and radial components separately in several different radial and vertical bins. We first determine the vertical gradient, and then 
$<[\mathrm{M} / \mathrm{H}]>$
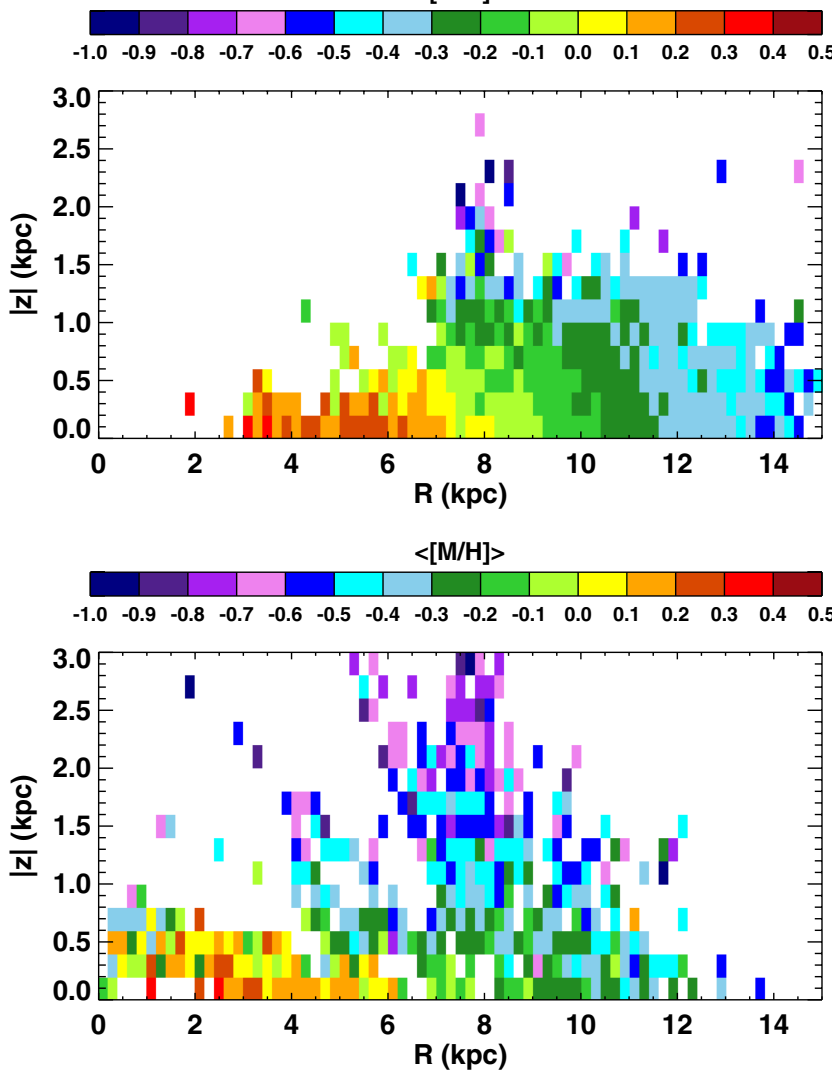

$<[\mathrm{M} / \mathrm{H}]>$
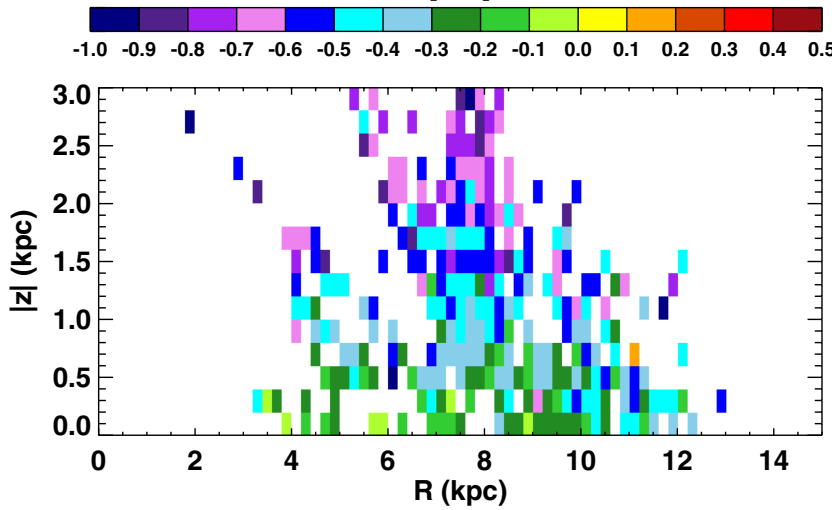

Figure 7. Top: the mean metallicity as a function of $\mathrm{R}$ and $|z|$ for low- $[\alpha / \mathrm{M}]$ populations and with $\log g>0.9$. The mean metallicity is higher in the plane of the Galaxy than it was for the entire sample, and the radial gradient is flatter in the inner Galaxy. Middle: the mean metallicity as a function of R and $|z|$ for high$[\alpha / \mathrm{M}]$ populations and with $\log g>0.9$. The mean metallicity is $0.2-0.3 \mathrm{dex}$ lower in the inner Galaxy than for the stars with solar $[\alpha / \mathrm{M}]$ abundance. Bottom: the mean metallicity as a function of $\mathrm{R}$ and $|z|$ for high- $[\alpha / \mathrm{M}]$ populations with $\log g>0.9$, but stars that have flagged $[\alpha / \mathrm{M}]$ abundance measurements (stars with $T_{\text {eff }}<4200 \mathrm{~K}$ ) are removed. We lose most stars in the inner Galaxy, but the mean metallicity in the rest of the Galaxy is relatively unchanged.

(A color version of this figure is available in the online journal.)

measure the radial gradient in a series of $|z|$ bins after correcting the stars to a common height $|\bar{z}|$, because the vertical gradient is significantly steeper than the radial one.

\subsubsection{Vertical Metallicity Gradient}

We measured vertical gradients from $0<|z|<2 \mathrm{kpc}$ in several radial bins between 1 and $15 \mathrm{kpc}$; we used $2 \mathrm{kpc}$ wide bins to ensure that there are significant numbers of stars at all

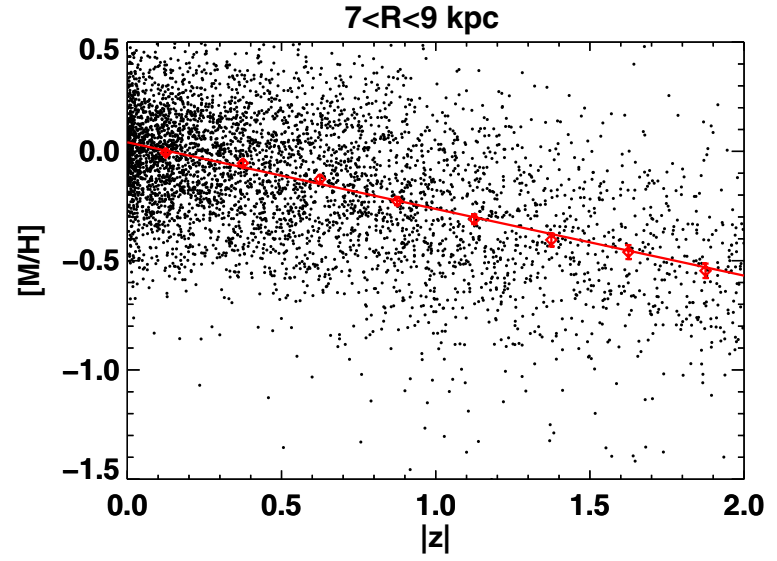

Figure 8. Vertical abundance gradient for the entire sample for stars at the solar circle, with the red diamonds representing the median metallicity in each $\Delta|z|=0.25 \mathrm{kpc}$ bin. The slope and intercept of the fit are reported in Table 1 . The vertical gradient is smoothly varying over the range of our sample, and no discontinuity (e.g., between a thick and thin disk) is found.

(A color version of this figure is available in the online journal.)

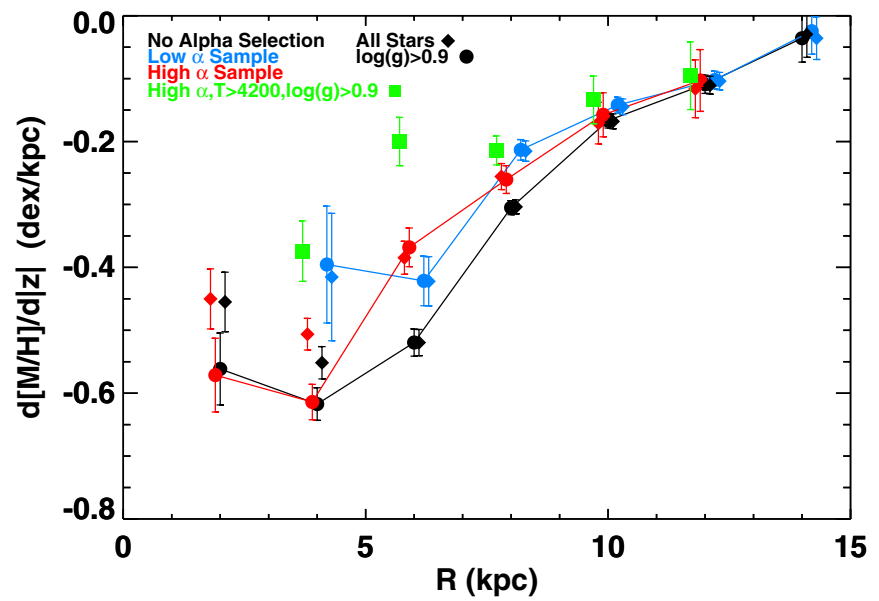

Figure 9. Vertical gradient $d[\mathrm{M} / \mathrm{H}] / d|z|$ for the APOGEE sample as a function of Galactocentric radius. Results are shown for the full sample (black) as well as for the $[\alpha / \mathrm{M}]$ selected subsamples (blue for low- $[\alpha / \mathrm{M}]$, red and green for high- $[\alpha / \mathrm{M}])$. The measured slopes and their associated errors are slightly offset in radius to allow them to be more easily seen in the figure. The vertical gradients are steeper at smaller Galactocentric radii. The vertical gradients are similar for both the low- and high- $[\alpha / \mathrm{M}]$ samples.

(A color version of this figure is available in the online journal.)

heights above the plane. The vertical gradient in each radial bin is measured by finding the median metallicity $[\mathrm{M} / \mathrm{H}]$ in $0.25 \mathrm{kpc}$ intervals in $|z|$ and determining a linear fit to the median values. Figure 8 displays an example for the bin with $7<R<9 \mathrm{kpc}$. The standard error in the median is adopted as the uncertainty on each median point; these uncertainties are quite small, given the large sample size. A linear fit to the median metallicities appears to provide a good representation of the data.

The derived linear vertical gradients $d[\mathrm{M} / \mathrm{H}] / d|z|$ as a function of Galactocentric radius are shown in Figure 9 for the entire sample, as well as for the $[\alpha / \mathrm{M}]$ selected subsamples. While results are shown for a $1<R<3 \mathrm{kpc}$ bin, the derived gradient in the innermost Galaxy could be affected by remaining biases against the most metal-rich stars, as well as the inclusion of stars from the bulge and/or bar.

The vertical gradient is a smoothly changing function of Galactocentric radius, becoming less steep at larger radii. 


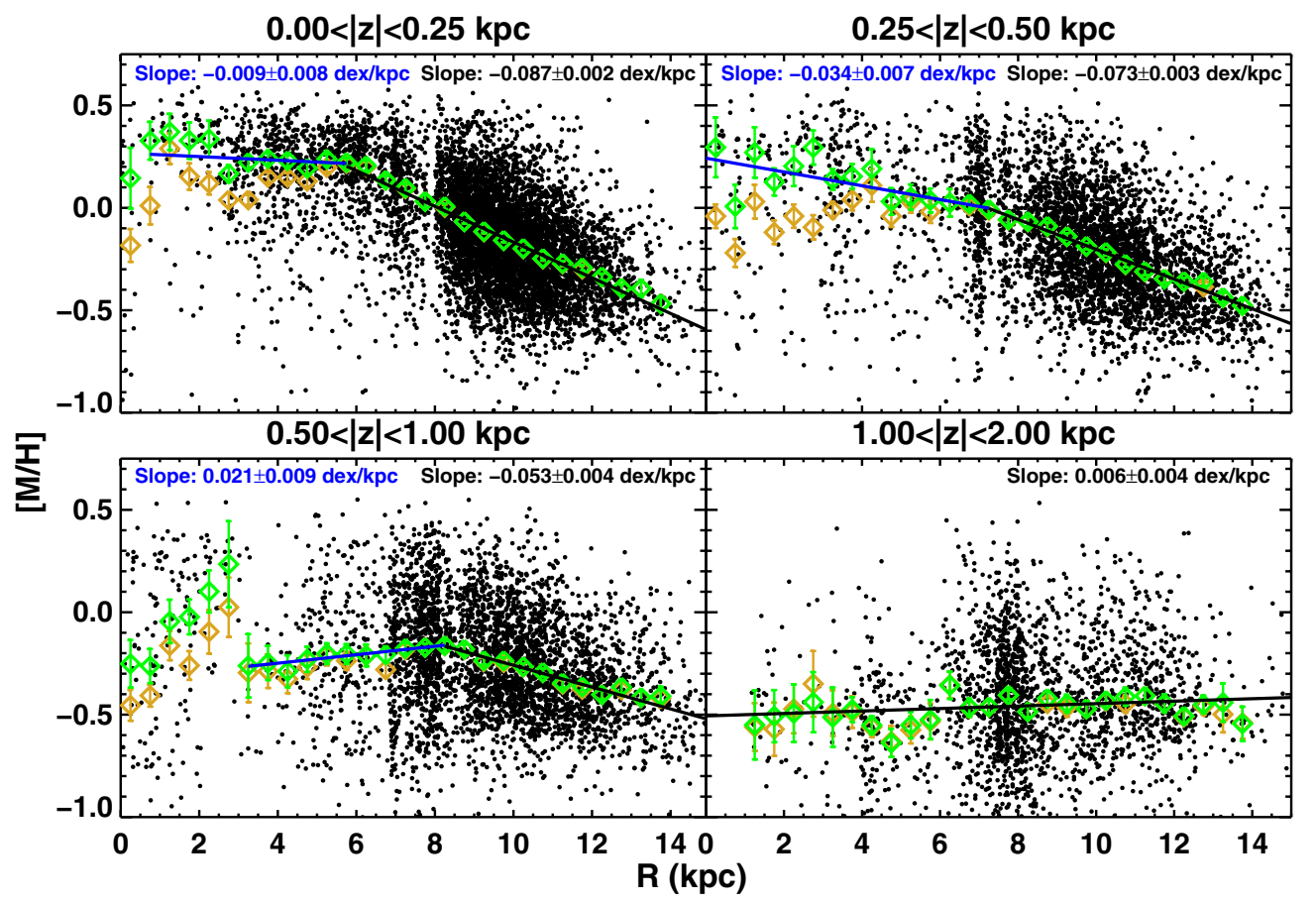

Figure 10. Observed radial metallicity gradients over a range of $|z|$ for our sample of stars with $\log g>0.9$. Green diamonds are the median metallicity for these stars, while gold diamonds are the median metallicity for the entire sample (i.e., including stars with $\log g<0.9$ ). The gradients presented in the text are fits to the green diamonds. We find the gradient is steepest in the plane of the Galaxy, and the slope decreases with height. In the inner Galaxy, the gradient becomes significantly less steep in the plane, with the transition at $R \sim 6 \mathrm{kpc}$.

(A color version of this figure is available in the online journal.)

Table 1

The Observed Vertical Gradients and Intercepts (at $z=0$ ) as a Function of $R$ for the Different Samples

\begin{tabular}{|c|c|c|c|c|c|c|c|c|}
\hline \multirow{2}{*}{$\begin{array}{l}\text { Radial Range } \\
(\mathrm{kpc})\end{array}$} & \multicolumn{2}{|c|}{ All Stars } & \multicolumn{2}{|c|}{ Low $\alpha$ Stars } & \multicolumn{2}{|c|}{ High $\alpha$ Stars } & \multicolumn{2}{|c|}{ High $\alpha$ Stars, $T_{\text {eff }}>4200 \mathrm{~K}$} \\
\hline & {$[\mathrm{M} / \mathrm{H}](z=0)$} & $d[\mathrm{M} / \mathrm{H}] / d|z|$ & {$[\mathrm{M} / \mathrm{H}](z=0)$} & $d[\mathrm{M} / \mathrm{H}] / d|z|$ & {$[\mathrm{M} / \mathrm{H}](z=0)$} & $d[\mathrm{M} / \mathrm{H}] / d|z|$ & {$[\mathrm{M} / \mathrm{H}](z=0)$} & $d[\mathrm{M} / \mathrm{H}] / d|z|$ \\
\hline $1<R<3$ & $0.39 \pm 0.04$ & $-0.56 \pm 0.06$ & & & $0.39 \pm 0.04$ & $-0.57 \pm 0.06$ & & \\
\hline $3<R<5$ & $0.31 \pm 0.02$ & $-0.62 \pm 0.03$ & $0.30 \pm 0.11$ & $-0.40 \pm 0.09$ & $0.28 \pm 0.02$ & $-0.61 \pm 0.03$ & $-0.04 \pm 0.20$ & $-0.37 \pm 0.05$ \\
\hline $5<R<7$ & $0.27 \pm 0.01$ & $-0.52 \pm 0.02$ & $0.27 \pm 0.03$ & $-0.42 \pm 0.04$ & $0.02 \pm 0.03$ & $-0.37 \pm 0.03$ & $-0.19 \pm 0.06$ & $-0.22 \pm 0.04$ \\
\hline $7<R<9$ & $0.04 \pm 0.01$ & $-0.31 \pm 0.01$ & $0.03 \pm 0.02$ & $-0.21 \pm 0.02$ & $-0.09 \pm 0.03$ & $-0.26 \pm 0.02$ & $-0.17 \pm 0.05$ & $-0.22 \pm 0.02$ \\
\hline $9<R<11$ & $-0.15 \pm 0.01$ & $-0.17 \pm 0.01$ & $-0.15 \pm 0.01$ & $-0.14 \pm 0.01$ & $-0.25 \pm 0.03$ & $-0.16 \pm 0.04$ & $-0.28 \pm 0.03$ & $-0.13 \pm 0.04$ \\
\hline $11<R<13$ & $-0.29 \pm 0.01$ & $-0.11 \pm 0.02$ & $-0.29 \pm 0.01$ & $-0.10 \pm 0.02$ & $-0.32 \pm 0.04$ & $-0.10 \pm 0.05$ & $-0.33 \pm 0.03$ & $-0.10 \pm 0.05$ \\
\hline $13<R<15$ & $-0.43 \pm 0.02$ & $-0.04 \pm 0.04$ & $-0.43 \pm 0.01$ & $-0.03 \pm 0.04$ & $\ldots$ & $\ldots$ & $\ldots$ & $\ldots$ \\
\hline
\end{tabular}

The vertical gradient is similar for all of the $[\alpha / \mathrm{M}]$ selected subsamples. For the entire sample, the vertical gradient is generally steeper than for either the low- or high- $[\alpha / \mathrm{M}]$ populations, because the low- $[\alpha / \mathrm{M}]$ population has a higher mean metallicity and shorter vertical scale height than the high- $[\alpha / \mathrm{M}]$ population (Bovy et al. 2012c).

Table 1 presents the linear fits to the mean metallicities, as a function of height above the plane, for several different zones of Galactocentric radius for both the full sample and for the $[\alpha / \mathrm{M}]$ selected subsamples. The vertical gradients are all well characterized by a single linear fit.

\subsubsection{Radial Metallicity Gradient}

We measure the radial gradient in four different ranges of $|z|: 0<|z|<0.25,0.25<|z|<0.5,0.5<|z|<1$, and $1<|z|<2 \mathrm{kpc}$ between $0<R<15 \mathrm{kpc}$. Because of the significant vertical gradient, there can be trends even within these bins (especially for the larger bins at larger distance from the plane), so we shift all stars to a common $|z|$ in each vertical bin by using the linear vertical abundance gradient fit to correct the metallicities of individual stars to the bin center. For each bin in $|z|$, the radial gradient is then computed by determining the median metallicity every $0.5 \mathrm{kpc}$ between $0<R<15 \mathrm{kpc}$. We use the standard error in the median to characterize the uncertainty in median metallicity in each radial bin. Figure 10 presents the radial gradients for entire APOGEE sample, while Figures 11 and 12 show the gradients for the low- and high- $[\alpha / \mathrm{M}]$ populations.

The gradients for the entire sample for $|z|<1 \mathrm{kpc}$ are not well-described by a single linear component. The slope is steeper in the outer Galaxy compared to the inner Galaxy, and the slope decreases with height above the plane. As previously noted, results for the innermost parts of the Galaxy could be influenced both by a remaining small bias against the most metal-rich stars and also because some of stars in the inner regions could come from a different structure component.

For low- $[\alpha / \mathrm{M}]$ populations the radial gradients have a break in the gradient for stars with $|z|<0.5 \mathrm{kpc}$ : the slope is flatter in the inner Galaxy compared to the outer Galaxy. For stars with $0.5<|z|<2 \mathrm{kpc}$, however, a single linear component describes the radial gradient, with a shallower slope. 


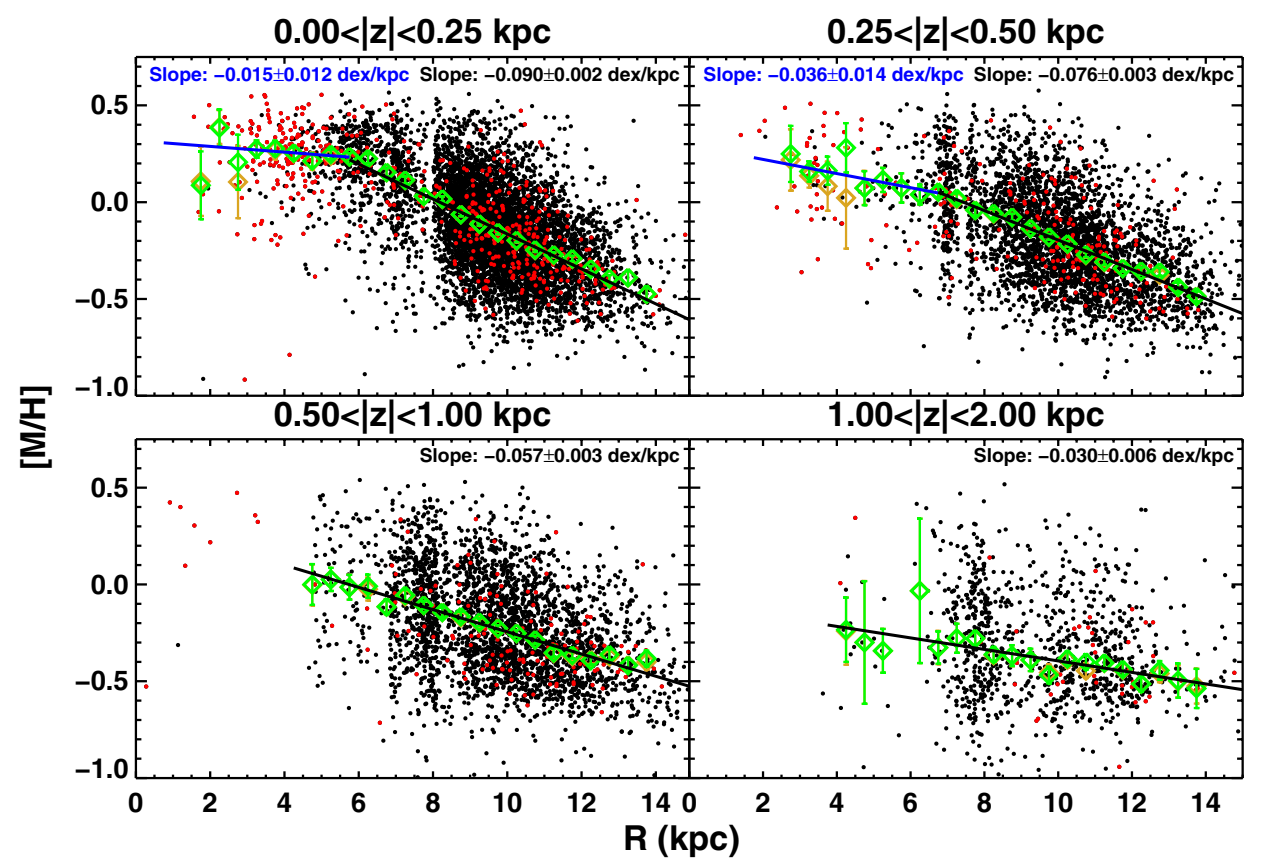

Figure 11. Observed radial metallicity gradients over a range of $|z|$ for low- $[\alpha / \mathrm{M}]$ populations, after metallicities were corrected for the observed vertical gradient. Black dots provide the most reliable and complete sample with $\log g>0.9$ and $T_{\text {eff }}>4200 \mathrm{~K}$; red dots are stars with $T_{\text {eff }}<4200 \mathrm{~K}$. The gold symbols are the same as in the previous figure. The results are similar compared to that for the entire sample shown in Figure 10: the slope decreases with height about the plane, and the slope is shallower in the inner Galaxy with a transition at $R \sim 6 \mathrm{kpc}$.

(A color version of this figure is available in the online journal.)

The high- $[\alpha / \mathrm{M}]$ populations are not well described by a single linear component for $|z|<0.5 \mathrm{kpc}$. However, it is possible that some of the high $\alpha$, high-metallicity stars with $\mathrm{R}<6 \mathrm{kpc}$ may be contaminated by a bulge population, and/or that the $[\alpha / \mathrm{M}]$ abundance determination is not accurate for these stars, many of which have effective temperatures less than $4000 \mathrm{~K}$. The discontinuity seen in the entire sample for $0.5<|z|<1 \mathrm{kpc}$ at $R \sim 4 \mathrm{kpc}$ is driven by high-[ $\alpha / \mathrm{M}]$ stars in the inner regions, which potentially belong to the bulge.

Table 2 lists the linear fits to the median metallicities as a function of Galactocentric radius for different heights above the plane for the full sample and for the $[\alpha / \mathrm{M}]$ selected subsamples. For the zones in which it appears that a single linear fit cannot represent the data, we present two-piece linear fits, and specify the break radius in the table. The quantitative fits are derived only in as far as a linear fit seems to represent the data; the range over which the fit is derived is shown by the extent of the line in Figure 10.

\subsection{Robustness of the Measured Gradients}

The observed scatter in metallicity of \pm 0.2 dex (at the one $\sigma$ level) observed in Figures 5, 8, 10, 11, and 12 at different locations in the Galaxy is real. If the shape of the MDF changes as a function of location, then the interpretation of gradients as determined from the median metallicity might need to be revised; we will investigate this issue in future work.

The small errors on the measured gradients result from the large sample size observed by APOGEE, since we adopt the standard error in the median for the uncertainty of the median metallicity in each bin. We also characterized the gradients and their errors using a jack-knife method (Efron \& Tibshirani 1993), which returned similar results (within $1 \sigma$ ) and uncertainties as the standard error of the median. We also performed linear fits to the raw metallicities (i.e., no binning) and recovered similar gradients.

\section{DISCUSSION}

\subsection{Comparison to Previous Literature: Mean Metallicities}

The APOGEE results extend previous measurements of mean metallicities to both smaller and larger Galactocentric radii, and with coverage at different heights above the plane. In particular, the ability of APOGEE to probe dust-obscured populations allows our sample to include stars in and near the Galactic plane at significant distances.

In regions covered by previous studies, we find similar mean metallicities. The mean metallicity of our low- $[\alpha / \mathrm{M}]$ sample close to the plane of the Galaxy is similar to the metallicities found by Luck et al. (2011) in their sample of Cepheids at all Galactocentric radii: they find mean metallicities of $0.25,0.1$, and -0.3 dex at $R$ of 6,8 , and $13 \mathrm{kpc}$ respectively, in good agreement with our measurements of $0.25,0.05$, and $-0.3 \mathrm{dex}$ for these radii. These measurements are also consistent with the mean metallicities observed by Frinchaboy et al. (2013; using APOGEE open clusters, which are distinct from the main survey targets considered here) and Yong et al. (2012) in their surveys of Galactic open clusters.

Away from the plane, Cheng et al. (2012b) measure a mean $[\mathrm{Fe} / \mathrm{H}]$ of -0.5 dex for stars with $|z|>1 \mathrm{kpc}$ using SDSS/ SEGUE observations, which is the same value that we measure for the APOGEE sample. For stars at the solar circle between $0.25<|z|<0.5 \mathrm{kpc}$, we measure a slightly higher mean metallicity, $\langle[\mathrm{M} / \mathrm{H}]\rangle=-0.1$, than Cheng et al. (2012b), who measure a mean $[\mathrm{Fe} / \mathrm{H}]$ of -0.2 dex.

\subsection{Vertical Metallicity Gradient}

The APOGEE data provide a first opportunity to characterize the vertical metallicity gradient over a large range of Galactocentric radii. As shown in Figure 9, a vertical gradient exists at all Galactocentric radii, but it becomes shallower at larger $R$; the radial gradients become flatter at larger $|z|$. 

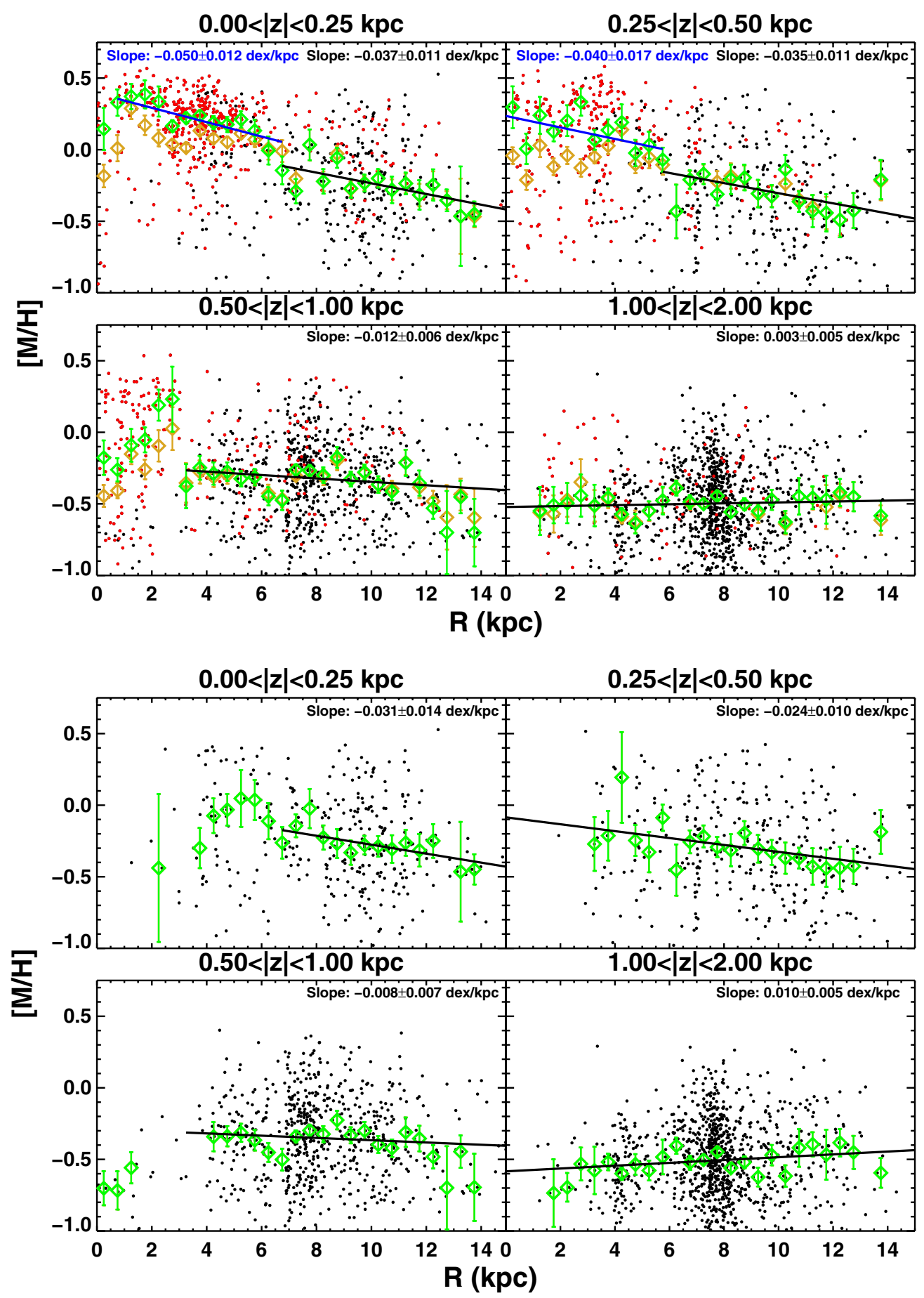

Figure 12. Top: same as Figure 11, but for high- $[\alpha / \mathrm{M}]$ subsamples. Bottom: similar to the top panel, but with stars cooler than $4200 \mathrm{~K}$ removed. The slope of the gradient is shallower for the $\alpha$-enhanced samples compared to that of the low- $[\alpha / \mathrm{M}]$ sample, becoming flat for $|z|>0.5 \mathrm{kpc}$.

(A color version of this figure is available in the online journal.)

Our measurements are fairly consistent with previous results where they overlap. We measure a gradient of $-0.213 \pm$ $0.016 \mathrm{dex} \mathrm{kpc}^{-1}$ for the low- $[\alpha / \mathrm{M}]$ sample at the solar circle. Previous studies for similar populations of stars measure slightly steeper slopes of $-0.295 \pm 0.005 \mathrm{dex} \mathrm{kpc}^{-1}$ (Chen et al. 2003), $-0.23 \pm 0.04 \mathrm{dex} \mathrm{kpc}^{-1}$ (Bartašiūtè et al. 2003), and $-0.29 \pm 0.06 \mathrm{dex} \mathrm{kpc}^{-1}$ (Marsakov \& Borkova 2006). However, other studies of open clusters have found little evidence for a vertical gradient (Carrera \& Pancino 2011; Jacobson et al. 2011a). One potential explanation for the discrepancy is that previous studies did not take into account the radial gradient when measuring the vertical gradient (Carrera \& Pancino 2011). Our results, however, do take the radial gradient into account and are not compatible with measurements from Carrera \& Pancino (2011). The open cluster sample is small, however, and has very few objects more than $0.5 \mathrm{kpc}$ above the plane, making this measurement heavily weighted by a few clusters. Additionally, the open cluster sample is dominated by objects with ages $<1 \mathrm{Gyr}$, so there are potentially age-metallicity effects between the two samples as well. 
Table 2

The Observed Radial Gradients as a Function of $|z|$ for the Different Subsamples

\begin{tabular}{|c|c|c|c|}
\hline \multicolumn{4}{|c|}{$0<|z|<0.25 \mathrm{kpc}$} \\
\hline Sample & Radial Range (kpc) & {$[\mathrm{M} / \mathrm{H}](R=6 \mathrm{kpc})$} & $d[\mathrm{M} / \mathrm{H}] / d R$ \\
\hline All stars & $0<R<5$ & $0.21 \pm 0.06$ & $-0.009 \pm 0.008$ \\
\hline All stars & $5<R<15$ & $0.18 \pm 0.02$ & $-0.087 \pm 0.002$ \\
\hline Low $\alpha$ stars & $0<R<5$ & $0.23 \pm 0.10$ & $-0.015 \pm 0.012$ \\
\hline Low $\alpha$ stars & $5<R<15$ & $0.20 \pm 0.02$ & $-0.090 \pm 0.002$ \\
\hline $\operatorname{High} \alpha$ stars & $0<R<6$ & $0.09 \pm 0.09$ & $-0.050 \pm 0.012$ \\
\hline High $\alpha$ stars & $6<R<15$ & $-0.09 \pm 0.13$ & $-0.037 \pm 0.011$ \\
\hline High $\alpha$ stars, $T_{\text {eff }}>4200 \mathrm{~K}$ & $6<R<15$ & $-0.19 \pm 0.17$ & $-0.024 \pm 0.014$ \\
\hline \multicolumn{4}{|c|}{$0.25<|z|<0.5 \mathrm{kpc}$} \\
\hline Sample & Radial Range (kpc) & {$[\mathrm{M} / \mathrm{H}](R=6 \mathrm{kpc})$} & $d[\mathrm{M} / \mathrm{H}] / d R$ \\
\hline All stars & $0<R<7$ & $0.04 \pm 0.06$ & $-0.034 \pm 0.007$ \\
\hline All stars & $7<R<15$ & $0.09 \pm 0.04$ & $-0.073 \pm 0.003$ \\
\hline Low $\alpha$ stars & $1<R<6$ & $0.08 \pm 0.12$ & $-0.036 \pm 0.014$ \\
\hline Low $\alpha$ stars & $6<R<15$ & $0.11 \pm 0.03$ & $-0.076 \pm 0.003$ \\
\hline $\operatorname{High} \alpha$ stars & $0<R<5$ & $-0.01 \pm 0.12$ & $-0.040 \pm 0.017$ \\
\hline High $\alpha$ stars & $5<R<15$ & $-0.16 \pm 0.12$ & $-0.035 \pm 0.011$ \\
\hline High $\alpha$ stars, $T_{\text {eff }}>4200 \mathrm{~K}$ & $0<R<15$ & $-0.23 \pm 0.10$ & $-0.024 \pm 0.010$ \\
\hline \multicolumn{4}{|c|}{$0.5<|z|<1 \mathrm{kpc}$} \\
\hline Sample & Radial Range (kpc) & {$[\mathrm{M} / \mathrm{H}](R=6 \mathrm{kpc})$} & $d[\mathrm{M} / \mathrm{H}] / d R$ \\
\hline All stars & $3<R<8$ & $-0.21 \pm 0.09$ & $0.021 \pm 0.009$ \\
\hline All stars & $8<R<15$ & $-0.05 \pm 0.05$ & $-0.053 \pm 0.004$ \\
\hline Low $\alpha$ stars & $4<R<15$ & $-0.02 \pm 0.04$ & $-0.057 \pm 0.003$ \\
\hline $\operatorname{High} \alpha$ stars & $3<R<15$ & $-0.30 \pm 0.06$ & $-0.012 \pm 0.006$ \\
\hline High $\alpha$ stars, $T_{\text {eff }}>4200 \mathrm{~K}$ & $3<R<15$ & $-0.33 \pm 0.07$ & $-0.007 \pm 0.007$ \\
\hline \multicolumn{4}{|c|}{$1<|z|<2 \mathrm{kpc}$} \\
\hline Sample & Radial Range (kpc) & {$[\mathrm{M} / \mathrm{H}](R=6 \mathrm{kpc})$} & $d[\mathrm{M} / \mathrm{H}] / d R$ \\
\hline All stars & $0<R<15$ & $-0.47 \pm 0.04$ & $0.006 \pm 0.004$ \\
\hline Low $\alpha$ stars & $3<R<15$ & $-0.28 \pm 0.08$ & $-0.030 \pm 0.006$ \\
\hline $\operatorname{High} \alpha \operatorname{stars}$ & $0<R<15$ & $-0.50 \pm 0.05$ & $0.003 \pm 0.005$ \\
\hline High $\alpha$ stars, $T_{\text {eff }}>4200 \mathrm{~K}$ & $0<R<15$ & $-0.53 \pm 0.05$ & $0.010 \pm 0.005$ \\
\hline
\end{tabular}

Notes. The intercept of the fits are reported for $R=6 \mathrm{kpc}$, which is the approximate radius at which the break occurs for most samples that have two components.

For the high- $[\alpha / \mathrm{M}]$ population we measure a gradient of $-0.26 \pm 0.022 \mathrm{dex} \mathrm{kpc}^{-1}$ at the solar circle, slightly higher than the slopes of $-0.22 \pm 0.07 \mathrm{dex} \mathrm{kpc}^{-1}$ observed by Chen et al. (2011) and $-0.22 \pm 0.03 \mathrm{dex} \mathrm{kpc}^{-1}$ by Ak et al. (2007) for their samples of thick-disk stars. Soubiran et al. (2008) measure a slope of $-0.31 \pm 0.03 \mathrm{dex} \mathrm{kpc}^{-1}$ for their sample of red clump giants that span a range of populations, in good agreement with our measurement of $-0.305 \pm 0.011 \mathrm{dex} \mathrm{kpc}^{-1}$ for the entire sample. Note that the measured gradient at $R=2 \mathrm{kpc}$ and $R=4 \mathrm{kpc}$ are the most likely to be affected by a metallicity bias. In Figure 9, this is shown by the difference in slope between the sample with $\log g>0.9$ and the sample with all stars included: the latter sample is affected by the bias against high-metallicity stars imposed by the ASPCAP spectral grid edge, which is reflected in the shallower vertical gradient for that sample. If there is still metallicity bias present in the sample, the measured slopes in the inner Galaxy may not be accurate, and are likely underestimated.

Interestingly, the vertical gradients appear to be similar for both low- and high- $[\alpha / \mathrm{M}]$ subsamples for $R>6 \mathrm{kpc}$. If the high- $[\alpha / \mathrm{M}]$ sample represents an older population of stars compared to low- $[\alpha / \mathrm{M}]$ sample, one might expect a different vertical gradient for these stars if the gradient is the result of the heating of a thin disk.
However, there is a significant difference in the vertical gradients at $R<6 \mathrm{kpc}$. Since the radial gradient in low- $[\alpha / \mathrm{M}]$ stars flattens more than it is does for higher- $[\alpha / \mathrm{M}]$ stars, the vertical gradient becomes significantly steeper for high- $[\alpha / \mathrm{M}]$ stars in the inner disk.

Several authors have found that older populations have larger scale heights, higher- $[\alpha / \mathrm{M}]$ ratios, and lower metallicity than younger populations (Lee et al. 2011; Bovy et al. 2012c; Schlesinger et al. 2012). Minchev et al. (2013) measure vertical gradients at the solar circle for their suite of simulations, in which populations of the same age show no vertical gradient; they find that observed vertical gradients in their simulations are due to mixing of young, metal-rich populations that dominate the stellar density in the plane with older, metal-poor populations that dominant the stellar density above the disk. If these results are true for the Milky Way, the observed flattening of the vertical gradient with radius suggests that the outer disk is more uniform in age, while the large vertical gradient in the inner Galaxy implies that a larger range of ages is present.

\subsection{Radial Metallicity Gradient}

The extended coverage of the APOGEE sample allows us to characterize the radial gradient over a large range of radii at various heights above the plane. 


\subsubsection{Inner Regions}

While we find that there is a negative radial metallicity gradient within a few $\mathrm{kpc}$ of the solar radius, this gradient appears to flatten in the inner regions of the galaxy, at $R \lesssim 6 \mathrm{kpc}$. This flattening appears to be more significant in the low- $[\alpha / \mathrm{M}]$ sample than it does in the high- $[\alpha / \mathrm{M}]$ sample. The change in slope from the outer to inner regions is also stronger closer to the plane of the Galaxy than away from it.

Some degree of mixing of stars might be expected as a result of the presence of a Galactic bar, and it is possible that our observed flattening of the gradient might be a sign of this effect. It is also possible that our results might be affected by some remaining bias against higher metallicity stars in the innermost regions and/or by the influence of stellar populations belonging to the bulge.

Our result is similar to that reported by Hill et al. (2012), who measured a sample of $\sim 200 \mathrm{RC}$ stars in the direction of the Galactic center, and found a median $[\mathrm{Fe} / \mathrm{H}]$ of 0.17 at $R \sim 6 \mathrm{kpc}$ and a flat radial gradient continuing to $R \sim 2 \mathrm{kpc}$. This is in excellent agreement with our measurements of a median $[\mathrm{M} / \mathrm{H}]$ of 0.18 for $R=6 \mathrm{kpc}$ (see Table 2 or Figure 10) and a roughly flat gradient in the inner Galaxy. However, their sample also may suffer from bias against metal-rich stars in the inner Galaxy $(R \lesssim 4 \mathrm{kpc})$.

\subsubsection{Intermediate Radii}

At $R \gtrsim 6 \mathrm{kpc}$, our measured radial gradient in the plane for low $[\alpha / \mathrm{M}]$ stars of $-0.090 \pm 0.002 \mathrm{dex} \mathrm{kpc}^{-1}$ is in excellent agreement with open cluster measurements from Frinchaboy et al. (2013), who find a slope of $-0.09 \pm 0.03 \mathrm{dex} \mathrm{kpc}^{-1}$. In that sample, all but two of the clusters lie within $0.25 \mathrm{kpc}$ of the plane. Nordström et al. (2004) reported a radial gradient of $-0.099 \pm 0.011 \mathrm{dex} \mathrm{kpc}^{-1}$ for the intermediate age populations observed in the GCS, again in good agreement with our measurement of $-0.090 \mathrm{dex} \mathrm{kpc}^{-1}$.

Considering the vertical dependence of radial gradients, we find similar trends for $R \gtrsim 6 \mathrm{kpc}$ as Cheng et al. (2012b): the gradients become flatter as one moves away from the plane. The gradient in the plane, $-0.087 \pm 0.002 \mathrm{dex} \mathrm{kpc}^{-1}$, is steeper than that found by the SEGUE team (Cheng et al. 2012b), who measure a slope of $-0.066_{-0.044}^{+0.030} \mathrm{dex} \mathrm{kpc}^{-1}$ in the plane; however, this result could partly arise from the fact that their sample was measured for stars with $0.15<|z|<0.25 \mathrm{kpc}$, i.e., it did not extend to $b=0^{\circ}$. Allende Prieto et al. (2006) found no discernible gradient for their sample of thick-disk stars between $1<|z|<3 \mathrm{kpc}$, consistent with our observations in our full sample for $|z|>1 \mathrm{kpc}$. As one moves away from the plane, the population becomes more metal-poor and less variable with Galactocentric radius.

Our large sample, along with the ability to split the sample by $[\alpha / \mathrm{M}]$, allows us to recognize that the radial gradient is steeper for low- $[\alpha / \mathrm{M}]$ stars than for higher- $[\alpha / \mathrm{M}]$ stars at all distances from the plane; even in our $1<|z|<2 \mathrm{kpc}$ bin, there are sufficient numbers of low- $[\alpha / \mathrm{M}]$ stars to demonstrate a negative radial gradient.

Although the measured radial gradients are much flatter for the high- $[\alpha / \mathrm{M}]$ population, our measurements demonstrate that this population behaves in much the same way as the low- $[\alpha / \mathrm{M}]$ population, with the radial gradient becoming flatter with height above the plane and the vertical gradient becoming steeper in the inner Galaxy.
If one interprets the high- $[\alpha / \mathrm{M}]$ sample as an older population, our data suggest that the radial gradient is steeper for younger stars than for older ones. However, while it is possible that this could result from a metallicity gradient in the gas that grows steeper with time, it could also result from the dilution of a pre-existing gradient with time as might occur, for example, if radial migration was important. There is disagreement in the literature over this result. Stanghellini \& Haywood (2010) observed planetary nebula across a range of ages and find that older populations have shallower metallicity gradients than younger populations. However, in a similar study of a different set of planetary nebula, Maciel \& Costa (2009) find the opposite result; they observed the gradient to flatten with time, with older populations having steeper gradients. Recent models of chemi$\mathrm{cal}$ and galaxy evolution predict that radial migration can wipe out gradients of older populations, causing younger populations to have steeper observed gradients (e.g., Roškar et al. 2008; Loebman et al. 2011; Kubryk et al. 2013). Our result of the steepening of the gradient with time are compatible with these models and the results from Stanghellini \& Haywood (2010).

\subsubsection{Outer Regions}

Frinchaboy et al. (2013) measured a single radial fit of $-0.09 \mathrm{dex} \mathrm{kpc}^{-1}$ for their sample of open clusters observed with APOGEE, but find that it is potentially better fit with two components: a steeper gradient of $-0.2 \mathrm{dex} \mathrm{kpc}^{-1}$ between $7.9<R<10 \mathrm{kpc}$, and a flat radial gradient for clusters with $R>10 \mathrm{kpc}$. Several previous studies (Costa et al. 2004; Jacobson et al. 2011b; Yong et al. 2012) have shown evidence for a transition to a flat radial gradient at large Galactocentric radii in the plane, with the transition occurring between 10 and $14 \mathrm{kpc}$. This flattening of the radial gradient is not observed with Cepheids (Luck et al. 2011; Luck \& Lambert 2011; Lemasle et al. 2013). There is some question about whether the apparent flattening might arise if one does not consider vertical gradients when the radial gradients are measured (Cheng et al. 2012b). Jacobson et al. (2011b) suggest that the transition radius to a flat gradient depends on the age of the population being studied: older open cluster populations show a transition to a flat gradient at $R \sim 10 \mathrm{kpc}$, while younger clusters show the transition at much larger radii, $R \sim 14 \mathrm{kpc}$. (Lemasle et al. 2013) find that the Cepheids in the outer disk are consistent with the result from Jacobson et al. (2011b), although the Cepheids from Luck $\&$ Lambert (2011) do not seem to be compatible with this result.

We see no significant evidence for any flattening out to $R \sim 14 \mathrm{kpc}$, after correcting for vertical gradients, except perhaps in the $0.5<|z|<1 \mathrm{kpc}$ bin. However, the DR10 data do not contain sufficient stars at larger radii to provide a good constraint on flattening at large radii. Future APOGEE observations may have a greater number of stars at large Galactocentric radii, allowing a more accurate determination of the behavior of the radial gradient in this area of the Galaxy.

Another possible explanation of the discrepancies found in previous studies is that the gas densities are much lower in the outer Galaxy and the chemical abundance is more sensitive to local events. The observations at large Galactocentric radii are sensitive to the population(s) and line(s) of sight being studied (Luck et al. 2011). With the uniform radial coverage of APOGEE, we hope that the extension of the data set beyond the first year data considered here will be useful for characterizing the gradient at large radii.

When we consider the $[\alpha / \mathrm{M}]$ subsamples, we find that the high- $\alpha$ population appears to truncate at $R \sim 10-12 \mathrm{kpc}$, 
as shown in Figures 7 and 12. Previous studies of the $\alpha$-enhanced population have found that it has a shorter scale length than the thin disk (Bensby et al. 2011; Cheng et al. 2012a). A detailed characterization of the APOGEE selection function is still in progress, so we cannot comment on the scale length of the $\alpha$-enhanced populations, but qualitatively, our observations are consistent with these previous studies.

\section{CONCLUSIONS}

We present mean metallicity maps and chemical abundance gradients observed in the first year of SDSS-III/APOGEE observations for a sample of nearly 20,000 red giants, and for subsamples that split the sample by $[\alpha / \mathrm{M}]$. Our primary results are as follows.

1. Radial gradients exist in the plane, but become flatter or non-existent above the plane.

2. The radial gradient appears to flatten at $R<6 \mathrm{kpc}$, more dramatically nearer the disk than away from it, and to a greater degree for low- $[\alpha / \mathrm{M}]$ stars.

3. Radial gradients are steeper for low- $[\alpha / \mathrm{M}]$ stars than they are for high- $[\alpha / \mathrm{M}]$ stars at all heights above the plane; a negative gradient exists for the low- $[\alpha / M]$ population even far from the plane, while the gradient for high- $[\alpha / M]$ stars disappears or even becomes slightly positive far from the plane.

4. Vertical metallicity gradients exist, but are flatter at larger Galactocentric radii.

5. The vertical gradients are similar for low- and high- $[\alpha / \mathrm{M}]$ subsamples at $R \gtrsim 6 \mathrm{kpc}$.

6. There is a real spread in the metallicities in all Galactic zones; the spread appears to be larger at smaller Galactocentric radii and at larger distances from the mid-plane.

Detailed modeling will be required to understand how these results can constrain models of disk formation and evolution, in particular, to understand the roles of the evolution of the radial and vertical structure of the disk, heating of disk populations, radial and vertical migration, the star formation history, and the importance of external events (mergers or disturbances).

APOGEE is an ongoing project, and future observations will greatly expand the sample size and potentially expand the coverage to larger radii and $|z|$. The data reported in this paper represent only a fraction of the expected SDSS-III sample size. A second phase of APOGEE observations in SDSS-IV is currently being planned and will further improve the coverage of the survey. Additionally, improvements are being made in the chemical abundance determinations from ASPCAP. We expect to be able to extend results to stars with $T_{\text {eff }}<3500$, to better understand the $\alpha$-element abundances for stars with $T_{\text {eff }}<4200$, and to measure abundances for $\sim 15$ separate elements with a goal of 0.1 dex accuracy. Different elements may have different gradients, and the addition of several new chemical species will provide more information on the chemical enrichment history of the Milky Way.

Funding for SDSS-III has been provided by the Alfred P. Sloan Foundation, the Participating Institutions, the National Science Foundation, and the U.S. Department of Energy Office of Science. The SDSS-III Web site is http://www.sdss3.org/. SDSS-III is managed by the Astrophysical Research Consortium for the Participating Institutions of the SDSS-III Collaboration including the University of Arizona, the Brazilian Participation Group, Brookhaven National Laboratory, Carnegie
Mellon University, University of Florida, the French Participation Group, the German Participation Group, Harvard University, the Instituto de Astrofisica de Canarias, the Michigan State/ Notre Dame/JINA Participation Group, Johns Hopkins University, Lawrence Berkeley National Laboratory, Max Planck Institute for Astrophysics, Max Planck Institute for Extraterrestrial Physics, New Mexico State University, New York University, Ohio State University, Pennsylvania State University, University of Portsmouth, Princeton University, the Spanish Participation Group, University of Tokyo, University of Utah, Vanderbilt University, University of Virginia, University of Washington, and Yale University.

We thank the anonymous referee for useful comments. M.H, J.H., and S.M, acknowledge support for this research from the National Science Foundation (AST-1109178). J.B. was supported by NASA through Hubble Fellowship grant HST-HF51285.01 from the Space Telescope Science Institute, which is operated by the Association of Universities for Research in Astronomy, Incorporated, under NASA contract NAS526555. K.C. acknowledges partial support for this research from the National Science Foundation (AST-0907873). G.Z. has been supported by an NSF Astronomy and Astrophysics Postdoctoral Fellowship under Award No. AST-1203017. T.C.B. acknowledges partial support for this work from grant PHY 08-22648: Physics Frontier Center/Joint Institute for Nuclear Astrophysics (JINA), awarded by the US National Science Foundation.

\section{REFERENCES}

Adelman-McCarthy, J. K., Agueros, M. A., Allam, S. S., et al. 2007, ApJS, 172,634

Adibekyan, V. Z., Sousa, S. G., Santos, N. C., et al. 2012, A\&A, 545, A32

Ahn, C. P., Alexandroff, R., Prieto, C. A., et al. 2013, ApJS, accepted (arXiv:1307.7735)

Aihara, H., Allende Prieto, C., An, D., et al. 2011, ApJS, 193, 29

Ak, S., Bilir, S., Karaali, S., Buser, R., \& Cabrera-Lavers, A. 2007, NewA, 12,605

Allende Prieto, C., Beers, T. C., Wilhelm, R., et al. 2006, AJ, 636, 804

Allende Prieto, C., Majewski, S. R., Cunha, K., et al. 2008, AN, 329, 4

Anders, F., Chiappini, C., Santiago, B. X., et al. 2013, A\&A, in press (arXiv:1311.4549)

Balser, D. S., Rood, R. T., Bania, T. M., \& Anderson, L. D. 2011, ApJ, 738,27

Bartašiūtè, S., Aslan, Z., Boyle, R. P., et al. 2003, BaltA, 12, 539

Benjamin, R. A., Churchwell, E., Babler, B. L., et al. 2003, PASP, 115, 953

Bensby, T., Alves-Brito, A., Oey, M. S., Yong, D., \& Meléndez, J. 2011, ApJL, 735, L46

Bird, J. C., Kazantzidis, S., Weinberg, D. H., et al. 2013, ApJ, 773, 43

Boeche, C., Siebert, A., Piffl, T., et al. 2013, A\&A, 559, A59

Bovy, J., Rix, H.-W., \& Hogg, D. W. 2012a, ApJ, 751, 131

Bovy, J., Rix, H.-W., Hogg, D. W., et al. 2012b, ApJ, 755, 115

Bovy, J., Rix, H.-W., Liu, C., et al. 2012c, ApJ, 753, 148

Bressan, A., Marigo, P., Girardi, L., et al. 2012, MNRAS, 427, 127

Burnett, B., \& Binney, J. 2010, MNRAS, 407, 16

Carrell, K., Chen, Y., \& Zhao, G. 2012, AJ, 144, 185

Carrera, R., \& Pancino, E. 2011, A\&A, 535, A30

Chen, L., Hou, J. L., \& Wang, J. J. 2003, AJ, 125, 1397

Chen, Y. Q., Zhao, G., Carrell, K., \& Zhao, J. K. 2011, AJ, 142, 184

Cheng, J. Y., Rockosi, C. M., Morrison, H. L., et al. 2012a, ApJ, 752, 51

Cheng, J. Y., Rockosi, C. M., Morrison, H. L., et al. 2012b, ApJ, 746, 149

Chiappini, C., Matteucci, F., \& Romano, D. 2001, AJ, 554, 1044

Churchwell, E., Babler, B. L., Meade, M. R., et al. 2009, PASP, 121, 213

Costa, R. D. D., Uchida, M. M. M., \& Maciel, W. J. 2004, A\&A, 423, 199

Daflon, S., \& Cunha, K. 2004, ApJ, 617, 1115

Daflon, S., Cunha, K., de la Reza, R., Holtzman, J., \& Chiappini, C. 2009, AJ, 138,1577

Efron, B., \& Tibshirani, R. 1993, An Introduction to the Bootstrap (Boca Raton, FL: CRC Press)

Eisenstein, D. J., Weinberg, D. H., Agol, E., et al. 2011, AJ, 142, 72

Fazio, G. G., \& Team, T. I. 2004, ApJS, 154, 7 
Frinchaboy, P. M., Thompson, B., Jackson, K. M., et al. 2013, ApJ, 777, 1 Genovali, K., Lemasle, B., Bono, G., et al. 2013, A\&A, 554, A132 Girardi, L., Groenewegen, M. A. T., Hatziminaoglou, E., \& Da Costa, L. 2005, A\&A, 436, 895

Girardi, L., \& Salaris, M. 2001, MNRAS, 323, 109

Gonzalez, O. A., Rejkuba, M., Zoccali, M., et al. 2013, A\&A, 552, A110

Gunn, J. E., Siegmund, W. A., \& Al, E. J. M. E. 2006, AJ, 131, 87

Hartkopf, W. I., \& Yoss, K. M. 1982, AJ, 87, 1679

Henry, R. B. C., Kwitter, K. B., Jaskot, A. E., et al. 2010, ApJ, 724, 748

Hill, V., Babusiaux, C., Gómez, A., et al. 2012, EPJWC, 19, 06001

Hou, J. L., Prantzos, N., \& Boissier, S. 2000, A\&A, 362, 921

Ivezic, Z., Sesar, B., Juric, M., et al. 2008, ApJ, 684, 40

Jacobson, H. R., Friel, E. D., \& Pilachowski, C. A. 2011a, AJ, 141, 58

Jacobson, H. R., Pilachowski, C. A., \& Friel, E. D. 2011b, AJ, 142, 59

Jurić, M., Ivezić, v., Brooks, A., Lupton, R. H., et al. 2008, ApJ, 673, 864

Kobayashi, C., \& Nakasato, N. 2011, ApJ, 729, 16

Kordopatis, G., Recio-Blanco, A., De Laverny, P., et al. 2011, A\&A, 535, A107

Kubryk, M., Prantzos, N., \& Athanassoula, E. 2013, MNRAS, 436, 1479

Larson, R. B. 1976, MNRAS, 176, 31

Lee, Y. S., Beers, T. C., An, D., et al. 2011, ApJ, 738, 187

Lemasle, B., François, P., Genovali, K., et al. 2013, A\&A, 558, A31

Loebman, S. R., Roškar, R., Debattista, V. P., et al. 2011, ApJ, 737, 8

Luck, R. E., Andrievsky, S. M., Kovtyukh, V. V., Gieren, W., \& Graczyk, D. 2011, AJ, 142, 51

Luck, R. E., \& Lambert, D. L. 2011, AJ, 142, 136
Maciel, W., \& Costa, R. 2009, in The Galaxy Disk in Cosmological Context (IAU Symp. 254), ed. J. Anderson, J. Bland-Hawthorn, \& B. Nordström (Cambridge: Cambridge Univ. Press), 38

Majewski, S. R., Zasowski, G., \& Nidever, D. L. 2011, ApJ, 739, 25

Marsakov, V. A., \& Borkova, T. V. 2006, AstL, 32, 376

Mészáros, S., Holtzman, J., García Pérez, A. E., et al. 2013, AJ, 146, 133

Minchev, I., Chiappini, C., \& Martig, M. 2013, A\&A, 558, A9

Minchev, I., Famaey, B., Quillen, A. C., et al. 2012, A\&A, 548, A127

Ness, M., Freeman, K., Athanassoula, E., et al. 2013, MNRAS, 430, 836

Nordström, B., Mayor, M., Andersen, J., et al. 2004, A\&A, 418, 989

Roškar, R., Debattista, V. P., Quinn, T. R., Stinson, G. S., \& Wadsley, J. 2008, ApJL, 684, L79

Santiago, B. X., Brauer, D. E., Anders, F., et al. 2014, A\&A, submitted

Schlesinger, K. J., Johnson, J. A., Rockosi, C. M., et al. 2012, ApJ, 761, 160

Schönrich, R., \& Binney, J. 2009, MNRAS, 396, 203

Sellwood, J. A., \& Binney, J. J. 2002, MNRAS, 336, 785

Soubiran, C., Bienaymé, O., Mishenina, T. V., \& Kovtyukh, V. V. 2008, A\&A, 480, 91

Stanghellini, L., \& Haywood, M. 2010, ApJ, 714, 1096

Steinmetz, M., Zwitter, T., Siebert, A., et al. 2006, AJ, 132, 1645

Wright, E. L., Eisenhardt, P. R. M., Mainzer, A., et al. 2010, AJ, 140, 22

Yanny, B., Rockosi, C., Newberg, H., et al. 2009, AJ, 137, 4377

Yong, D., Carney, B. W., \& Friel, E. D. 2012, AJ, 144, 95

York, D. G. 2000, AJ, 120, 9

Zasowski, G., Johnson, J. A., Frinchaboy, P. M., et al. 2013, AJ, 146, 81

Zoccali, M., Hill, V., Lecureur, A., et al. 2008, A\&A, 486, 177 